\title{
Weak turbulence in dusty plasmas with collisional dust charging: Quasilinear wave-particle interaction
}

\author{
Renato A. Galvão* and Luiz F. Ziebell \\ Instituto de Física, UFRGS, Caixa Postal 15051, 91501-970 Porto Alegre, RS, Brasil \\ (Received 10 April 2015; revised manuscript received 10 July 2015; published 13 August 2015)
}

\begin{abstract}
Starting from the exact microscopic equations for an unmagnetized dusty plasma, where the dust charge is regarded as a new degree of freedom of the system, we present a self-consistent set of equations that is suitable for weak turbulence analyses, where we have considered that the dust is electrically charged by absorption of plasma particles through inelastic collisions. In the quasilinear limit of the wave-particle interaction, we find a modified equation for the spectral energy density of the plasma electric field, and two other equations of the spectral energy densities associated with the fluctuation of the dust distribution due to plasma particle absorption, and with the fluctuation of the dust charging current. Different dispersion relations are found respectively for each type of oscillation. The corresponding kinetic equations for the waves are given, as well as the temporal evolution equations of the dust and plasma particles.
\end{abstract}

DOI: 10.1103/PhysRevE.92.023102

\section{INTRODUCTION}

The presence of dust may greatly modify the electromagnetic properties of a plasma. This feature relies on the fact that dust particles are in general significantly more massive and bigger than ions and electrons and as such can act as a sink of plasma particles through inelastic collisions. The collisional absorption of new charged particles by a dust grain is, to a high degree, influenced by the charges already attached to its surface in a self-consistent process where the electric interaction between plasma particles and dust particles plays an important role. Other factors also contribute to the collisional charging process, for instance, the distributions of dust size and density and the mean thermal energy of plasma particles. In addition, mechanisms of emission of particles can also be effective, as photoemission and secondary emission of electrons. Therefore, as a consequence of the dust presence, the propagation of electrostatic and electromagnetic waves in dusty plasmas exhibits new features compared with dustless plasmas. Many investigations have been made on this subject, and here we cite a few authors [1-5], who use either a magnetohydrodynamic or a kinetic approach. Besides the dust charge, the dust mass is particularly important and is intrinsically related with two low-frequency normal modes of propagation in unmagnetized weakly coupled dusty plasmas, the dust acoustic and the dust ion-acoustic modes [6], which were theoretically predicted by Rao, Shukla, and Yu [7] and Shukla and Silin [8].

The eigenmodes that arise from the collective motions in a plasma may become unstable and may also interact one with another. If the amplitudes of the eigenmodes are sufficiently small, their interaction will be weak, but not necessarily negligible, and can be classified into three different classes. The first one is the quasilinear wave-particle interaction, which occurs more strongly when the resonance $\omega=\mathbf{k} \cdot \mathbf{v}$ is satisfied. The second one, the nonlinear three-wave interaction, satisfies $\omega_{1}+\omega_{2}=\omega_{3}, \mathbf{k}_{1}+\mathbf{k}_{2}=\mathbf{k}_{3}$. The third one is the nonlinear wave-particle class, where the resonance condition occurs for

\footnotetext{
*galvaora@gmail.com

${ }^{\dagger}$ luiz.ziebell@ufrgs.br
}

PACS number(s): 52.25.Dg, 52.27.Lw, 52.35.Ra

$\omega_{1}-\omega_{2}=\left(\mathbf{k}_{1}-\mathbf{k}_{2}\right) \cdot \mathbf{v}$, corresponding to a wave scattering by a particle [9].

The theory which incorporates the description of these low-order nonlinear processes is the so-called weak turbulence theory, whose theoretical foundations were largely developed in the 1960s and 1970s [9-13] and have been revisited in more recent years [14-16]. The framework of weak turbulence theory has been employed to solve fundamental problems in plasma physics, since it provides tools for the understanding of nonlinear phenomena. Among the large number of papers which apply weak turbulence theory, we cite only a few as example [17-24]. In these papers the interested reader can find references to other publications on the subject. Weak turbulence theory also has been applied to investigate some nonlinear effects on dusty plasmas eigenmodes. For instance, Yi, Ryu, and Yoon have investigated the nonlinear frequency shift in coherent dust acoustic and dust ion-acoustic waves in an unmagnetized dusty plasma, caused by higher dust acoustic and dust ion-acoustic turbulence, respectively $[25,26]$.

The present work develops considering the dust charge as a variable quantity [27] in the context of weak turbulence theory. We have started from the exact microscopic distributions for dust particles and plasma electrons and ions, considering the dust charge as a new degree of freedom of the system, and considering the first-order kinetic equations in the Bogoliubov-Born-Green-Kirkwood-Yvon (BBGKY) hierarchy for a dusty plasma [28]. Among the diverse possible dust charging mechanisms we have considered for simplicity only the charging due to the absorption of plasma particles by inelastic collisions. The interaction between dust particles was also neglected for simplicity. This approximation is valid for investigations in dusty plasmas where the intergrain average distance is greater than the Debye length, $r_{d}>\lambda_{D}$, a condition typically found in plasma laboratory experiments and in diverse space plasma environments [29].

In analogy with the standard weak turbulence theory of dustless plasmas, we have expanded the plasma particles fluctuations and the dust particles fluctuations in powers of the plasma electric field, as well as in powers of the fields associated to the dust electric charging process. In the present work we concentrate the analysis on the effect of wave-particle 
interactions, obtaining a complete set of quasilinear equations which interconnects the time evolution of the averaged particle distribution functions with the kinetic equations for the actual wave fields and for fieldlike quantities which are related to dust charging fluctuations. A group of three independent dispersion relations naturally arises from such a set of equations and can provide relevant properties of electrostatic waves in an unmagnetized dusty plasma. The outcome is a self-consistent quasilinear kinetic formulation for the time evolution of plasma particles, electrostatic waves, and dust particles charged by collisional processes, which was not yet available in the literature, to best of our knowledge.

The work is structured as follows: In Sec. II we make a review of the kinetic description for a dusty plasma in the assumed configuration, introduce the relevant fluctuating quantities, and obtain a system of equations that is suitable for a weak turbulence analysis. In Sec. III the system of equations is expressed in terms of spectral variables for the fluctuating quantities. In Sec. IV we examine with further detail a set of kinetic equations which conduct us to a quasilinear analysis of the evolution of the dust-plasma system, taking into account electrostatic fluctuations. The associated spectral energy densities and corresponding dispersion relations are introduced. In Sec. $\mathrm{V}$ an example of application of the formalism is given, with detailed expressions of the quasilinear systems of equations for the case of a dusty plasma with a single ion species and a single electron population. Finally, we present our conclusions and final remarks in Sec. VI.

\section{KINETIC DESCRIPTION}

The possibility of absorption of a plasma particle by a dust grain can be modeled by redefining the microscopic particle distribution in the form [28]

$$
N_{\sigma}(\mathbf{X}, t)=\sum_{i=1}^{N_{\sigma}} \delta\left[\mathbf{X}-\mathbf{X}_{i \sigma}(t)\right] \theta\left(t_{i \sigma}-t\right),
$$

where $\mathbf{X} \equiv(\mathbf{r}, \mathbf{v}), \mathbf{X}_{i \sigma}(t) \equiv\left[\mathbf{r}_{i \sigma}(t), \mathbf{v}_{i \sigma}(t)\right]$ are six-dimensional vectors, $t_{i \sigma}$ is the instant of collision of the $i$ th particle with a dust grain, and $\theta(x)$ is the Heaviside step function. From (1), the equation for the time development of the distribution $N_{\sigma}$ can be written as

$$
\begin{aligned}
\frac{\partial N_{\sigma}}{\partial t} & +\frac{\partial N_{\sigma}}{\partial \mathbf{r}} \cdot \mathbf{v}+\frac{\partial N_{\sigma}}{\partial \mathbf{v}} \cdot \frac{\mathbf{F}_{\sigma}^{m}}{m_{\sigma}} \\
= & -\int d \mathcal{X}^{\prime} \Omega\left(\mathbf{r}-\mathbf{r}^{\prime}, \mathbf{v}-\mathbf{v}^{\prime}, a\right) N_{d}\left(\mathcal{X}^{\prime}, t\right) N_{\sigma}(\mathbf{X}, t),
\end{aligned}
$$

where $\mathcal{X} \equiv(\mathbf{r}, \mathbf{v}, q)$ are the coordinates in the dust sevendimension phase space, and the microscopic dust distribution is given as

$$
N_{d}(\mathcal{X}, t)=\sum_{j=1}^{N_{d}} \delta\left[\mathcal{X}-\mathcal{X}_{j}(t)\right],
$$

and the function

$$
\begin{aligned}
& \Omega\left(\mathbf{r}-\mathbf{r}^{\prime}, \mathbf{v}-\mathbf{v}^{\prime}, a\right) \\
& \quad=\delta\left(\left|\mathbf{r}-\mathbf{r}^{\prime}\right|-a\right) \theta\left[-\left(\mathbf{v}-\mathbf{v}^{\prime}\right) \cdot \mathbf{e}_{\mathbf{r}-\mathbf{r}^{\prime}}\right]\left|\left(\mathbf{v}-\mathbf{v}^{\prime}\right) \cdot \mathbf{e}_{\mathbf{r}-\mathbf{r}^{\prime}}\right|
\end{aligned}
$$

has dimension of frequency.
The microscopic equation for the dust component, disregarding the dust to dust interaction, is [28]

$$
\begin{aligned}
\frac{\partial N_{d}}{\partial t} & +\frac{\partial N_{d}}{\partial \mathbf{r}} \cdot \mathbf{v}+\frac{\partial N_{d}}{\partial \mathbf{v}} \cdot \frac{\mathbf{F}_{d}^{m}}{m_{d}} \\
= & -\sum_{\sigma} \int d \mathbf{X}^{\prime} N_{\sigma}\left(\mathbf{X}^{\prime}, t\right)\left[\Omega\left(\mathbf{r}-\mathbf{r}^{\prime}, \mathbf{v}-\mathbf{v}^{\prime}, a\right) N_{d}(\mathcal{X}, t)\right. \\
& -\Omega\left(\mathbf{r}-\mathbf{r}^{\prime}, \mathbf{v}-\delta \mathbf{v}_{\sigma}-\mathbf{v}^{\prime}, a\right) \\
& \left.\times N_{d}\left(\mathbf{r}, \mathbf{v}-\delta \mathbf{v}_{\sigma}, q-q_{\sigma}, t\right)\right]
\end{aligned}
$$

The $q_{\sigma}$ symbol refers to the electric charge of the plasma species, and $\delta \mathbf{v}_{\sigma}$ is the change in the velocity of a dust grain due to a collision with a plasma particle. Since these are small parameters in the interaction, one can expand the right-hand side of Eq. (5), such that

$$
\frac{\partial N_{d}}{\partial t}+\frac{\partial N_{d}}{\partial \mathbf{r}} \cdot \mathbf{v}+\frac{\partial N_{d}}{\partial \mathbf{v}} \cdot \frac{\mathbf{F}_{d}^{m}}{m_{d}}=-\frac{\partial}{\partial q}\left[I^{m} N_{d}(\mathcal{X}, t)\right],
$$

where

$$
I^{m}=\sum_{\sigma} q_{\sigma} \int d \mathbf{X}^{\prime} N_{\sigma}\left(\mathbf{X}^{\prime}, t\right) \Omega\left(\mathbf{r}-\mathbf{r}^{\prime}, \mathbf{v}-\mathbf{v}^{\prime}, a\right) .
$$

The first-order term that remains in Eq. (6) is the term due to dust charging. The approximation in which the dust charge discreteness can be neglected is valid for sufficiently large dust grains, with a radius of order $a \sim 10^{-5} \mathrm{~cm} \mathrm{[30].}$

Averaging Eqs. (2) and (6),

$$
\begin{gathered}
\frac{\partial f_{\sigma}}{\partial t}+\frac{\partial f_{\sigma}}{\partial \mathbf{r}} \cdot \mathbf{v}+\left\langle\frac{\partial N_{\sigma}}{\partial \mathbf{v}} \cdot \frac{\mathbf{F}_{\sigma}^{m}}{m_{\sigma}}\right\rangle \\
=-\int d \mathcal{X}^{\prime} \Omega\left\langle N_{d}\left(\mathcal{X}^{\prime}, t\right) N_{\sigma}(\mathbf{X}, t)\right\rangle, \\
\frac{\partial f_{d}}{\partial t}+\frac{\partial f_{d}}{\partial \mathbf{r}} \cdot \mathbf{v}+\left\langle\frac{\partial N_{d}}{\partial \mathbf{v}} \cdot \frac{\mathbf{F}_{d}^{m}}{m_{d}}\right\rangle=-\left\langle\frac{\partial}{\partial q}\left(I^{m} N_{d}(\mathcal{X}, t)\right)\right\rangle,
\end{gathered}
$$

where

$$
\begin{aligned}
\left\langle N_{\sigma}(\mathbf{X}, t)\right\rangle= & \sum_{i=1}^{N_{\sigma}} \int d \mathbf{X}_{1 \sigma} \ldots \mathbf{X}_{N_{\sigma}} \delta\left[\mathbf{X}-\mathbf{X}_{i \sigma}(t)\right] \\
& \times \theta\left(t_{i \sigma}-t\right) G\left(\mathbf{X}_{1 \sigma} \ldots \mathbf{X}_{N_{\sigma}}\right)=f_{\sigma}(\mathbf{X}, t),
\end{aligned}
$$

which is nonzero for $t<t_{1 \sigma}$, and $G$ is the density of probability that at time $t$ the plasma particles are in the state $\left[\mathbf{X}_{1 \sigma}(t) \ldots \mathbf{X}_{N_{\sigma}}(t) ; t\right]$. From here to the end of this section we denote $\Omega\left(\mathbf{r}-\mathbf{r}^{\prime}, \mathbf{v}-\mathbf{v}^{\prime}, a\right)$ simply as $\Omega$.

Solutions to Eqs. (8) and (9) require a kinetic equation for the binary correlation $\left\langle N_{d}\left(\mathcal{X}^{\prime}, t\right) N_{\sigma}(\mathbf{X}, t)\right\rangle$, which in turn depends on equations of higher-order correlations, and so on. The sequence of steps results in a complete BBGKY hierarchy for a dusty plasma, as found in Ref. [28]. However, it is well known from plasma kinetic theory that such a chain of equations can be closed at some order, according to parameters of interest.

Taking the above into account, we introduce the fluctuation of the phase density $\delta N=N-\langle N\rangle$, which leads us to the replacement $\left\langle N_{d} N_{\sigma}\right\rangle \rightarrow\left\langle\delta N_{d} \delta N_{\sigma}\right\rangle+\left\langle N_{d}\right\rangle\left\langle N_{\sigma}\right\rangle$ and to the need of a binary correlation for the fluctuations $\left\langle\delta N_{d} \delta N_{\sigma}\right\rangle$. At this point, weak turbulence starts to play a role, as it will become clear as the formalism further develops along the text, 
using the premise that the small parameter in the interaction can be taken as the ratio between the energy density of the fluctuating fields and the mean energy density of the plasma particles.

Using the definition of $I^{m}$ in Eq. (7), the equation for the dust distribution can be written as

$$
\begin{aligned}
\frac{\partial f_{d}}{\partial t} & +\frac{\partial f_{d}}{\partial \mathbf{r}} \cdot \mathbf{v}+\left\langle\frac{\partial N_{d}}{\partial \mathbf{v}} \cdot \frac{\mathbf{F}_{d}^{m}}{m_{d}}\right\rangle \\
= & -I(\mathbf{X}, t) \frac{\partial}{\partial q} f_{d}(\mathcal{X}, t)-\frac{\partial}{\partial q}\left\langle\delta I^{m}(\mathbf{X}, t) \delta N_{d}(\mathcal{X}, t)\right\rangle,
\end{aligned}
$$

where the following quantities with dimension of current have been defined:

$$
\begin{gathered}
I(\mathbf{X}, t)=\sum_{\sigma} q_{\sigma} \int d \mathbf{X}^{\prime} \Omega f_{\sigma}\left(\mathbf{X}^{\prime}, t\right), \\
\delta I^{m}(\mathbf{X}, t)=\sum_{\sigma} q_{\sigma} \int d \mathbf{X}^{\prime} \Omega \delta N_{\sigma}\left(\mathbf{X}^{\prime}, t\right) .
\end{gathered}
$$

Similarly,

$$
\begin{aligned}
\frac{\partial f_{\sigma}}{\partial t} & +\frac{\partial f_{\sigma}}{\partial \mathbf{r}} \cdot \mathbf{v}+\left\langle\frac{\partial N_{\sigma}}{\partial \mathbf{v}} \cdot \frac{\mathbf{F}_{\sigma}^{m}}{m_{\sigma}}\right\rangle \\
= & -\int d \mathcal{X}^{\prime} \Omega\left[f_{d}\left(\mathcal{X}^{\prime}, t\right) f_{\sigma}(\mathbf{X}, t)+\left\langle\delta N_{d}\left(\mathcal{X}^{\prime}, t\right) \delta N_{\sigma}(\mathbf{X}, t)\right\rangle\right]
\end{aligned}
$$

With the absence of magnetic fields, we only consider the electrostatic Maxwell's equations, where the divergence equation shall include the contribution of the dust component, such that

$$
\begin{aligned}
\frac{\partial}{\partial \mathbf{r}} \cdot \mathbf{E}^{m}(\mathbf{r}, t)= & 4 \pi\left[\sum_{\sigma} q_{\sigma} \int d \mathbf{v} N_{\sigma}(\mathbf{X}, t)\right. \\
& \left.+\int d q q \int d \mathbf{v} N_{d}(\mathcal{X}, t)\right]
\end{aligned}
$$

and, besides, $\partial / \partial \mathbf{r} \times \mathbf{E}^{m}=0$.

Neglecting any other kind of force, the acting force over a plasma particle is purely electric, $\mathbf{F}_{\sigma}^{m}=q_{\sigma} \mathbf{E}^{m}$. Considering that the electric field can also be written as an average value and a fluctuation, Eq. (13) is given as follows:

$$
\begin{aligned}
\frac{\partial f_{\sigma}}{\partial t} & +\frac{\partial f_{\sigma}}{\partial \mathbf{r}} \cdot \mathbf{v}+\frac{q_{\sigma}}{m_{\sigma}} \mathbf{E} \cdot \frac{\partial f_{\sigma}}{\partial \mathbf{v}} \\
= & -\frac{q_{\sigma}}{m_{\sigma}}\left\langle\frac{\partial \delta N_{\sigma}}{\partial \mathbf{v}} \cdot \delta \mathbf{E}^{m}\right\rangle-\frac{q_{\sigma}}{m_{\sigma} v_{*}}\left\langle\delta N_{\sigma}(\mathbf{X}, t) \delta E_{\sigma}(\mathbf{X}, t)\right\rangle \\
& -\omega_{\sigma}(\mathbf{X}, t) f_{\sigma}(\mathbf{X}, t),
\end{aligned}
$$

where we have introduced quantities $\delta E_{\sigma}$ and $\omega_{\sigma}$ with dimensions of electric field and frequency, respectively,

$$
\begin{gathered}
\delta E_{\sigma}(\mathbf{X}, t)=\frac{m_{\sigma} v_{*}}{q_{\sigma}} \int d \mathcal{X}^{\prime} \Omega \delta N_{d}\left(\mathcal{X}^{\prime}, t\right), \\
\omega_{\sigma}(\mathbf{X}, t)=\int d \mathcal{X}^{\prime} \Omega f_{d}\left(\mathcal{X}^{\prime}, t\right)
\end{gathered}
$$

The velocity $v_{*}$ was introduced as a characteristic velocity of the problem to be examined. For instance, in the case of electrostatic waves, it may be convenient to choose the thermal velocity of the electrons. The quantity $\delta E_{\sigma}$ is an effective field associated to dust fluctuations due to the absorption of plasma particles of type $\sigma$, while $\omega_{\sigma}$ is the average collision frequency.

For the dust component we obtain

$$
\begin{aligned}
\frac{\partial f_{d}}{\partial t}+ & \frac{\partial f_{d}}{\partial \mathbf{r}} \cdot \mathbf{v}+\frac{q}{m_{d}} \mathbf{E} \cdot \frac{\partial f_{d}}{\partial \mathbf{v}} \\
= & -\frac{q}{m_{d}}\left\langle\frac{\partial \delta N_{d}}{\partial \mathbf{v}} \cdot \delta \mathbf{E}^{m}\right\rangle-I(\mathbf{X}, t) \frac{\partial}{\partial q} f_{d}(\mathcal{X}, t) \\
& -\frac{\partial}{\partial q}\left\langle\delta I^{m}(\mathbf{X}, t) \delta N_{d}(\mathcal{X}, t)\right\rangle .
\end{aligned}
$$

Subtracting Eq. (15) from Eq. (2), one obtains an equation for the fluctuation of the distribution of particles of species $\sigma$,

$$
\begin{aligned}
\frac{\partial \delta N_{\sigma}}{\partial t} & +\frac{\partial \delta N_{\sigma}}{\partial \mathbf{r}} \cdot \mathbf{v}+\frac{q_{\sigma}}{m_{\sigma}} \frac{\partial f_{\sigma}}{\partial \mathbf{v}} \cdot \delta \mathbf{E}^{m} \\
= & -\frac{q_{\sigma}}{m_{\sigma}} \frac{\partial \delta N_{\sigma}}{\partial \mathbf{v}} \cdot \delta \mathbf{E}^{m}+\frac{q_{\sigma}}{m_{\sigma}}\left\langle\frac{\partial \delta N_{\sigma}}{\partial \mathbf{v}} \cdot \delta \mathbf{E}^{m}\right\rangle \\
& -\frac{q_{\sigma}}{m_{\sigma} v_{*}}\left[\delta N_{\sigma}(\mathbf{X}, t) \delta E_{\sigma}(\mathbf{X}, t)-\left\langle\delta N_{\sigma}(\mathbf{X}, t) \delta E_{\sigma}(\mathbf{X}, t)\right\rangle\right. \\
& \left.+f_{\sigma}(\mathbf{X}, t) \delta E_{\sigma}(\mathbf{X}, t)\right]+\omega_{\sigma}(\mathbf{X}, t) \delta N_{\sigma}(\mathbf{X}, t),
\end{aligned}
$$

where we have considered the average electric field $\mathbf{E}=0$.

Subtracting Eq. (18) from Eq. (6),

$$
\begin{aligned}
\frac{\partial \delta N_{d}}{\partial t} & +\frac{\partial \delta N_{d}}{\partial \mathbf{r}} \cdot \mathbf{v}+\frac{q}{m_{d}} \frac{\partial f_{d}}{\partial \mathbf{v}} \cdot \delta \mathbf{E}^{m} \\
= & -\frac{q}{m_{d}} \frac{\partial \delta N_{d}}{\partial \mathbf{v}} \cdot \delta \mathbf{E}^{m}+\left\langle\frac{q}{m_{d}} \frac{\partial \delta N_{d}}{\partial \mathbf{v}} \cdot \delta \mathbf{E}^{m}\right\rangle \\
& -\delta I^{m} \frac{\partial}{\partial q} \delta N_{d}+\frac{\partial}{\partial q}\left\langle\delta I^{m}(\mathbf{X}, t) \delta N_{d}(\mathcal{X}, t)\right\rangle \\
& -I \frac{\partial}{\partial q} \delta N_{d}-\delta I^{m} \frac{\partial}{\partial q} f_{d} .
\end{aligned}
$$

The set of equations for a complete dynamical description of the plasma is finally closed with the equation for the fluctuating plasma electric field,

$$
\begin{aligned}
& \frac{\partial}{\partial \mathbf{r}} \cdot \delta \mathbf{E}^{m}(\mathbf{r}, t) \\
& \quad=4 \pi\left(\sum_{\sigma} q_{\sigma} \int d \mathbf{v} \delta N_{\sigma}+\int d q q \int d \mathbf{v} \delta N_{d}\right) .
\end{aligned}
$$

Now we introduce a simplification by averaging the newly introduced quantities $\delta E_{\sigma}$ and $\omega_{\sigma}$ given by Eqs. (16) and (17) over the velocity variable and quantities $I$ and $\delta I^{m}$ given by Eqs. (11) and (12) over velocity and dust charge variable. As a result, the time evolution of the dusty plasma, considering only the occurrence of electrostatic waves, will be ruled by Eq. (21) and by Eqs. (15) and (18)-(20), where the fluctuating fields $\delta \mathbf{E}^{m}$, as well as the quantities $\omega_{\sigma}, \delta E_{\sigma}, I, \delta I^{m}$, are only 
a function of position and time,

$$
\begin{gathered}
\omega_{\sigma}(\mathbf{r}, t)=\frac{1}{n_{\sigma}} \int d \mathbf{v} f_{\sigma} \int d \mathcal{X}^{\prime} \Omega f_{d}\left(\mathcal{X}^{\prime}, t\right), \\
\delta E_{\sigma}(\mathbf{r}, t)=\frac{m_{\sigma} v_{*}}{n_{\sigma} q_{\sigma}} \int d \mathbf{v} f_{\sigma} \int d \mathcal{X}^{\prime} \Omega \delta N_{d}\left(\mathcal{X}^{\prime}, t\right), \\
I(\mathbf{r}, t)=\frac{1}{n_{d}} \int d \mathbf{v} d q f_{d} \sum_{\sigma} q_{\sigma} \int d \mathbf{X}^{\prime} \Omega f_{\sigma}\left(\mathbf{X}^{\prime}, t\right) \\
\delta I^{m}(\mathbf{r}, t)=\frac{1}{n_{d}} \int d \mathbf{v} d q f_{d} \sum_{\sigma} q_{\sigma} \int d \mathbf{X}^{\prime} \Omega \delta N_{\sigma}\left(\mathbf{X}^{\prime}, t\right)
\end{gathered}
$$

The averaged distributions are normalized such that $n_{\sigma}(\mathbf{r}, t)=$ $\int d \mathbf{v} f_{\sigma}(\mathbf{X}, t)$ and $n_{d}(\mathbf{r}, t)=\int d \mathbf{v} d q f_{d}(\mathcal{X}, t)$ are the number of plasma particles and dust particles per unit volume.

\section{SPECTRAL VARIABLES}

Since we are interested in the study of wave-particle interactions, it seems natural to work in the reciprocal spectral space, where we are led by performing a Fourier-Laplace transform of the relevant equations. To simplify the notation, in what follows we drop the $\delta$ symbol and the index $m$.

Initially, starting from (21), we obtain an equation for the Fourier transform of the fluctuations of the plasma electric field,

$$
E_{\mathbf{k}}(t)=-\frac{4 \pi i}{k}\left(\sum_{\sigma} q_{\sigma} \int d \mathbf{v} N_{\mathbf{k}}^{\sigma}+\int d q q \int d \mathbf{v} N_{\mathbf{k}}^{d}\right),
$$

where $k=|\mathbf{k}|$.

In the sequence, we consider the field given by Eq. (23),

$$
E_{\sigma}(\mathbf{r}, t)=\frac{m_{\sigma} v_{*}}{n_{\sigma} q_{\sigma}} \int d \mathbf{v} f_{\sigma} \int d \mathcal{X}^{\prime} \Omega\left(\mathbf{r}-\mathbf{r}^{\prime}, \mathbf{v}-\mathbf{v}^{\prime}, a\right) N_{d}\left(\mathcal{X}^{\prime}, t\right)
$$

Fourier transforming (27),

$$
\begin{aligned}
E_{\mathbf{k}}^{\sigma}(t)= & -\frac{4 \pi i}{k} \frac{q_{\sigma}}{n_{\sigma}} \int d \mathbf{v} f_{\sigma} \\
& \times \int d \mathbf{v}^{\prime} d q^{\prime} \Omega_{\mathbf{k}}^{\sigma}\left(\mathbf{v}-\mathbf{v}^{\prime}, a\right) N_{\mathbf{k}}^{d}\left(\mathbf{v}^{\prime}, q^{\prime}, t\right),
\end{aligned}
$$

where we have defined the nondimensional function

$$
\Omega_{\mathbf{k}}^{\sigma}\left(\mathbf{v}-\mathbf{v}^{\prime}, a\right)=(2 \pi)^{3} i \frac{n_{\sigma} v_{*} k}{\omega_{p \sigma}^{2}} \Omega_{\mathbf{k}}\left(\mathbf{v}-\mathbf{v}^{\prime}, a\right),
$$

where $\omega_{p \sigma}$ is the plasma frequency and the transforms of $\Omega$ and $N_{d}$ are given by

$$
\begin{aligned}
\Omega_{\mathbf{k}}\left(\mathbf{v}-\mathbf{v}^{\prime}, a\right) & =\frac{1}{(2 \pi)^{3}} \int d \mathbf{r} \Omega\left(\mathbf{r}, \mathbf{v}-\mathbf{v}^{\prime}, a\right) e^{-i \mathbf{k} . \mathbf{r}}, \\
N_{\mathbf{k}}^{d}\left(\mathbf{v}^{\prime}, q^{\prime}, t\right) & =\frac{1}{(2 \pi)^{3}} \int d \mathbf{r} N_{d}\left(\mathbf{r}, \mathbf{v}^{\prime}, q^{\prime}, t\right) e^{-i \mathbf{k} . \mathbf{r}} .
\end{aligned}
$$

We then proceed with Eq. (25),

$$
\begin{aligned}
I_{\mathbf{k}}(t)= & -\frac{4 \pi i}{k} \sum_{\sigma} q_{\sigma} \frac{q_{\sigma}^{2}}{m_{\sigma} v_{*}} \frac{1}{n_{d}} \int d \mathbf{v} d q f_{d} \\
& \times \int d \mathbf{v}^{\prime} \Omega_{\mathbf{k}}^{\sigma}\left(\mathbf{v}-\mathbf{v}^{\prime}, a\right) N_{\mathbf{k}}^{\sigma}\left(\mathbf{v}^{\prime}, t\right) .
\end{aligned}
$$

By assuming that distributions $f_{\sigma}$ and $f_{d}$ are uniform in space, we consider that quantities which depend on an integral form upon these distributions are also independent of position. Therefore, Eqs. (22) and (24) become

$$
\begin{aligned}
\omega_{\sigma}(t)= & \frac{1}{n_{\sigma}} \int d \mathbf{v} f_{\sigma} \int d \mathbf{r}^{\prime} d \mathbf{v}^{\prime} d q^{\prime} \\
& \times\left|\left(\mathbf{v}-\mathbf{v}^{\prime}\right) \cdot \mathbf{e}_{\mathbf{r}^{\prime}}\right| \delta\left(r^{\prime}-a\right) f_{d}\left(\mathbf{v}^{\prime}, q^{\prime}, t\right), \\
I(t)= & \frac{1}{n_{d}} \int d \mathbf{v} d q f_{d} \sum_{\sigma} q_{\sigma} \int d \mathbf{r}^{\prime} d \mathbf{v}^{\prime} \\
& \times \delta\left(r^{\prime}-a\right)\left|\left(\mathbf{v}-\mathbf{v}^{\prime}\right) \cdot \mathbf{e}_{\mathbf{r}^{\prime}}\right| f_{\sigma}\left(\mathbf{v}^{\prime}, t\right) .
\end{aligned}
$$

The equations which result from the transformations of the equations for the average distribution functions, and for the equations describing the evolution of the fluctuating distributions $N_{\sigma}$ and $N_{d}$, may be said to evolve in different time scales. In fact, it is possible to perform a multiple-time-scale expansion in the small parameter of the nonlinear interaction, $\varepsilon<1$, by extending the number of time variables such that $[11,13]$

$$
t=\varepsilon^{-1} t^{\prime}, \quad t^{\prime}=\varepsilon^{-1} t^{\prime \prime}, \quad t^{\prime \prime}=\varepsilon^{-1} t^{\prime \prime \prime} \ldots
$$

The slow oscillation time scale affects the plasma particles and dust averaged distributions, while the fast oscillation time scale is relevant for the fluctuations. Therefore, writing the fluctuating quantities as functions of $\left(t, t^{\prime}\right)$, and taking the Laplace transform over the fast time, the set of kinetic equations which we have obtained is transformed into the following:

$$
\begin{aligned}
& \frac{\partial f_{\sigma}\left(\mathbf{v}, t^{\prime}\right)}{\partial t^{\prime}}=-\omega_{\sigma}\left(t^{\prime}\right) f_{\sigma}\left(t^{\prime}\right)-\frac{q_{\sigma}}{m_{\sigma}} \int d \mathbf{n} \int d \mathbf{n}^{\prime}\left[\frac{\mathbf{k}^{\prime}}{k^{\prime}} \cdot \frac{\partial}{\partial \mathbf{v}}\left\langle N_{\mathbf{n}}^{\sigma}\left(t^{\prime}\right) E_{\mathbf{n}^{\prime}}\left(t^{\prime}\right)\right\rangle-\frac{1}{v_{*}}\left\langle N_{\mathbf{n}}^{\sigma}\left(t^{\prime}\right) E_{\mathbf{n}^{\prime}}^{\sigma}\left(t^{\prime}\right)\right\rangle\right] e^{i\left(\mathbf{k}+\mathbf{k}^{\prime}\right) \cdot \mathbf{r}-i\left(\omega+\omega^{\prime}\right) t}, \\
& \frac{\partial f_{d}\left(\mathbf{v}, q, t^{\prime}\right)}{\partial t^{\prime}}=-I\left(t^{\prime}\right) \frac{\partial f_{d}\left(t^{\prime}\right)}{\partial q}-\frac{q}{m_{d}} \int d \mathbf{n} \int d \mathbf{n}^{\prime}\left[\frac{\mathbf{k}^{\prime}}{k^{\prime}} \cdot \frac{\partial}{\partial \mathbf{v}}\left\langle N_{\mathbf{n}}^{d}\left(t^{\prime}\right) E_{\mathbf{n}^{\prime}}\left(t^{\prime}\right)\right\rangle-\frac{\partial}{\partial q}\left\langle N_{\mathbf{n}}^{d}\left(t^{\prime}\right) I_{\mathbf{n}^{\prime}}\left(t^{\prime}\right)\right\rangle\right] e^{i\left(\mathbf{k}+\mathbf{k}^{\prime}\right) \cdot \mathbf{r}-i\left(\omega+\omega^{\prime}\right) t,} \\
&-i\left(\omega+i \frac{\partial}{\partial t^{\prime}}-\mathbf{k} \cdot \mathbf{v}+i \omega_{\sigma}\right) N_{\mathbf{n}}^{\sigma}\left(t^{\prime}\right)=-\frac{q_{\sigma}}{m_{\sigma}} \frac{\partial f_{\sigma}}{\partial \mathbf{v}} \cdot \frac{\mathbf{k}}{k} E_{\mathbf{n}}\left(t^{\prime}\right)-\frac{q_{\sigma}}{m_{\sigma} v_{*}} f_{\sigma} E_{\mathbf{n}}^{\sigma}\left(t^{\prime}\right)-\int d \mathbf{n}^{\prime}\left(\frac{q_{\sigma}}{m_{\sigma}} \frac{\partial N_{\mathbf{n}-\mathbf{n}^{\prime}}^{\sigma}}{\partial \mathbf{v}} \cdot \frac{\mathbf{k}^{\prime}}{k^{\prime}} E_{\mathbf{n}^{\prime}}\left(t^{\prime}\right)\right. \\
&\left.-\frac{q_{\sigma}}{m_{\sigma}}\left\langle\frac{\partial N_{\mathbf{n}-\mathbf{n}^{\prime}}^{\sigma}}{\partial \mathbf{v}} \cdot \frac{\mathbf{k}^{\prime}}{k^{\prime}} E_{\mathbf{n}^{\prime}}\left(t^{\prime}\right)\right\rangle\right)-\frac{q_{\sigma}}{m_{\sigma} v_{*}} \int d \mathbf{n}^{\prime}\left(E_{\mathbf{n}^{\prime}}^{\sigma}\left(t^{\prime}\right) N_{\mathbf{n}-\mathbf{n}^{\prime}}^{\sigma}-\left\langle E_{\mathbf{n}^{\prime}}^{\sigma}\left(t^{\prime}\right) N_{\mathbf{n}-\mathbf{n}^{\prime}}^{\sigma}\right),\right.
\end{aligned}
$$




$$
\begin{gathered}
\left(-i \omega+\frac{\partial}{\partial t^{\prime}}+i \mathbf{k} \cdot \mathbf{v}+I \frac{\partial}{\partial q}\right) N_{\mathbf{n}}^{d}\left(t^{\prime}\right)=-\frac{q}{m_{d}} \frac{\partial f_{d}}{\partial \mathbf{v}} \cdot \frac{\mathbf{k}}{k} E_{\mathbf{n}}\left(t^{\prime}\right)-I_{\mathbf{n}}\left(t^{\prime}\right) \frac{\partial f_{d}}{\partial q}-\frac{\partial}{\partial q} \int d \mathbf{n}^{\prime}\left[I_{\mathbf{n}^{\prime}}\left(t^{\prime}\right) N_{\mathbf{n}-\mathbf{n}^{\prime}}^{d}-\left\langle I_{\mathbf{n}^{\prime}}\left(t^{\prime}\right) N_{\mathbf{n}-\mathbf{n}^{\prime}}^{d}\right)\right] \\
\quad-\int d \mathbf{n}^{\prime}\left(\frac{q}{m_{d}} \frac{\partial N_{\mathbf{n}-\mathbf{n}^{\prime}}^{d}}{\partial \mathbf{v}} \cdot \frac{\mathbf{k}^{\prime}}{k^{\prime}} E_{\mathbf{n}^{\prime}}\left(t^{\prime}\right)-\frac{q}{m_{d}}\left\langle\frac{\partial N_{\mathbf{n}-\mathbf{n}^{\prime}}^{d}}{\partial \mathbf{v}} \cdot \frac{\mathbf{k}^{\prime}}{k^{\prime}} E_{\mathbf{n}^{\prime}}\left(t^{\prime}\right)\right\rangle\right), \\
I_{\mathbf{n}}\left(t^{\prime}\right)=-\frac{4 \pi i}{k} \sum_{\sigma} q_{\sigma} \frac{q_{\sigma}^{2}}{m_{\sigma} v_{*}} \frac{1}{n_{d}} \int d \mathbf{v} d q f_{d} \int d \mathbf{v}^{\prime} \Omega_{\mathbf{k}}^{\sigma}\left(\mathbf{v}-\mathbf{v}^{\prime}, a\right) N_{\mathbf{n}}^{\sigma}\left(\mathbf{v}^{\prime}, t^{\prime}\right), \\
I\left(t^{\prime}\right)=\frac{1}{n_{d}} \int d \mathbf{v} d q f_{d} \sum_{\sigma} q_{\sigma} \int d \mathbf{r}^{\prime} d \mathbf{v}^{\prime} \delta\left(r^{\prime}-a\right)\left|\left(\mathbf{v}-\mathbf{v}^{\prime}\right) \cdot \mathbf{e}_{\mathbf{r}^{\prime}}\right| f_{\sigma}\left(\mathbf{v}^{\prime}, t^{\prime}\right), \\
\omega_{\sigma}\left(t^{\prime}\right)=\frac{1}{n_{\sigma}} \int d \mathbf{v} f_{\sigma} \int d \mathbf{r}^{\prime} d \mathbf{v}^{\prime} d q^{\prime}\left|\left(\mathbf{v}-\mathbf{v}^{\prime}\right) \cdot \mathbf{e}_{\mathbf{r}^{\prime}}\right| \delta\left(r^{\prime}-a\right) f_{d}\left(\mathbf{v}^{\prime}, q^{\prime}, t^{\prime}\right), \\
E_{\mathbf{n}}^{\sigma}\left(t^{\prime}\right)=-\frac{4 \pi i}{k} \frac{q_{\sigma}}{n_{\sigma}} \int d \mathbf{v} f_{\sigma} \int d \mathbf{v}^{\prime} d q^{\prime} \Omega_{\mathbf{k}}^{\sigma}\left(\mathbf{v}-\mathbf{v}^{\prime}, a\right) N_{\mathbf{n}}^{d}\left(\mathbf{v}^{\prime}, q^{\prime}, t^{\prime}\right), \\
E_{\mathbf{n}}\left(t^{\prime}\right)=-\frac{4 \pi i}{k}\left[\sum_{\sigma} q_{\sigma} \int d \mathbf{v} N_{\mathbf{n}}^{\sigma}\left(t^{\prime}\right)+\int d q q \int d \mathbf{v} N_{\mathbf{n}}^{d}\left(t^{\prime}\right)\right],
\end{gathered}
$$

where

$$
A_{\mathbf{k}}\left(t, t^{\prime}\right)=\int_{L} d \omega A_{\mathbf{n}}\left(t^{\prime}\right) \exp (-i \omega t), \quad A_{\mathbf{n}}\left(t^{\prime}\right)=\frac{1}{2 \pi} \int_{0}^{\infty} d t A_{\mathbf{k}}\left(t, t^{\prime}\right) \exp (i \omega t),
$$

for $A_{\mathbf{k}}\left(t, t^{\prime}\right)$ representing $N_{\mathbf{k}}^{\sigma}\left(t, t^{\prime}\right), N_{\mathbf{k}}^{d}\left(t, t^{\prime}\right), E_{\mathbf{k}}\left(t, t^{\prime}\right), E_{\mathbf{k}}^{\sigma}\left(t, t^{\prime}\right)$, and $I_{\mathbf{k}}\left(t, t^{\prime}\right)$ and where we have made $\mathbf{n}=(\mathbf{k}, \omega)$.

Introducing the operators

$$
\mathbf{g}_{\mathbf{n}}^{\sigma}(\mathbf{v})=-\frac{q_{\sigma}}{m_{\sigma}} \frac{\partial / \partial \mathbf{v}}{\omega-\mathbf{k} \cdot \mathbf{v}+i \omega_{\sigma}}
$$

and

$$
h_{\mathbf{n}}^{\sigma}(\mathbf{v})=-\frac{q_{\sigma}}{m_{\sigma}} \frac{v_{*}^{-1}}{\omega-\mathbf{k} \cdot \mathbf{v}+i \omega_{\sigma}},
$$

which contain the effect of the collision frequency $\omega_{\sigma}$, Eq. (36) can be written as

$$
N_{\mathbf{n}}^{\sigma}=i\left(\mathbf{g}_{\mathbf{n}}^{\sigma} \cdot \frac{\mathbf{k}}{k}\right) E_{\mathbf{n}} f_{\sigma}+i h_{\mathbf{n}}^{\sigma} E_{\mathbf{n}}^{\sigma} f_{\sigma}+i \int d \mathbf{n}^{\prime}\left[\mathbf{g}_{\mathbf{n}}^{\sigma}\left(N_{\mathbf{n}-\mathbf{n}^{\prime}}^{\sigma} \cdot \frac{\mathbf{k}^{\prime}}{k^{\prime}} E_{\mathbf{n}^{\prime}}-\left\langle N_{\mathbf{n}-\mathbf{n}^{\prime}}^{\sigma} \cdot \frac{\mathbf{k}^{\prime}}{k^{\prime}} E_{\mathbf{n}^{\prime}}\right\rangle\right)+h_{\mathbf{k} \omega}^{\sigma}\left(E_{\mathbf{n}^{\prime}}^{\sigma} N_{\mathbf{n}-\mathbf{n}^{\prime}}^{\sigma}-\left\langle E_{\mathbf{n}^{\prime}}^{\sigma} N_{\mathbf{n}-\mathbf{n}^{\prime}}^{\sigma}\right\rangle\right)\right] .
$$

The corresponding Eq. (37) for $N_{\mathbf{n}}^{d}$ requires a previous treatment of the operator $\partial / \partial q$, before it can be put in a form similar to (45). For this, we assume that there is a frequency $\omega_{d}$ such that

$$
I \frac{\partial N_{\mathbf{n}}^{d(1)}}{\partial q}=\omega_{d} N_{\mathbf{n}}^{d(1)}
$$

where $N_{\mathbf{k} \omega}^{d(1)}$ is the approximation in first order for $N_{\mathbf{k} \omega}^{d}$ given by Eq. (56). Then we obtain

$$
N_{\mathbf{n}}^{d}=i\left(\mathbf{g}_{\mathbf{n}}^{d} \cdot \frac{\mathbf{k}}{k}\right) E_{\mathbf{n}} f_{d}+i h_{\mathbf{n}}^{d} \hat{E}_{\mathbf{n}} f_{d}+i \int d \mathbf{n}^{\prime}\left[h_{\mathbf{n}}^{d}\left(\hat{E}_{\mathbf{n}^{\prime}} N_{\mathbf{n}-\mathbf{n}^{\prime}}^{d}-\left\langle\hat{E}_{\mathbf{n}^{\prime}} N_{\mathbf{n}-\mathbf{n}^{\prime}}^{d}\right\rangle\right)+\mathbf{g}_{\mathbf{n}}^{d}\left(N_{\mathbf{n}-\mathbf{n}^{\prime}}^{d} \cdot \frac{\mathbf{k}^{\prime}}{k^{\prime}} E_{\mathbf{n}^{\prime}}-\left\langle N_{\mathbf{n}-\mathbf{n}^{\prime}}^{d} \cdot \frac{\mathbf{k}^{\prime}}{k^{\prime}} E_{\mathbf{n}^{\prime}}\right\rangle\right)\right],
$$

where

$$
\begin{aligned}
\mathbf{g}_{\mathbf{n}}^{d}(\mathbf{v}, q) & =-\frac{q}{m_{d}} \frac{\partial / \partial \mathbf{v}}{\omega-\mathbf{k} \cdot \mathbf{v}+i \omega_{d}}, \\
h_{\mathbf{n}}^{d}(\mathbf{v}) & =-\frac{e^{2}}{m_{d}} \frac{v_{*}^{-1} \partial / \partial q}{\omega-\mathbf{k} \cdot \mathbf{v}+i \omega_{d}},
\end{aligned}
$$

and where we have defined the field

$$
\hat{E}_{\mathbf{n}}=\frac{m_{d} v_{*}}{e^{2}} I_{\mathbf{n}}
$$

in terms of the fluctuating current $I_{\mathbf{n}}$, which is represented by Eq. (38). The quantity $\hat{E}_{\mathbf{n}}$ may be regarded as the component of an effective field associated to fluctuations of the current over the dust particles.

Equation (45) can be expanded as a series of powers of the amplitudes of the electric fields,

$$
N_{\mathbf{n}}^{\sigma}=\sum_{j=1}^{\infty} N_{\mathbf{n}}^{\sigma(j)} .
$$


RENATO A. GALVÃO AND RUIZ F. ZIEBELL

PHYSICAL REVIEW E 92, 023102 (2015)

At first order, the equation is simply

$$
N_{\mathbf{n}}^{\sigma(1)}=i\left(\mathbf{g}_{\mathbf{n}}^{\sigma} \cdot \frac{\mathbf{k}}{k}\right) E_{\mathbf{n}} f_{\sigma}+i h_{\mathbf{n}}^{\sigma} E_{\mathbf{n}}^{\sigma} f_{\sigma}
$$

and at $j$ th order we have the formal expression

$$
N_{\mathbf{n}}^{\sigma(j)}=i \int d \mathbf{n}^{\prime}\left[\mathbf{g}_{\mathbf{n}}^{\sigma}\left(N_{\mathbf{n}-\mathbf{n}^{\prime}}^{\sigma(j-1)} \cdot \frac{\mathbf{k}^{\prime}}{k^{\prime}} E_{\mathbf{n}^{\prime}}-\left\langle N_{\mathbf{n}-\mathbf{n}^{\prime}}^{\sigma(j-1)} \cdot \frac{\mathbf{k}^{\prime}}{k^{\prime}} E_{\mathbf{n}^{\prime}}\right\rangle\right)+h_{\mathbf{n}}^{\sigma}\left(E_{\mathbf{n}^{\prime}}^{\sigma} N_{\mathbf{n}-\mathbf{n}^{\prime}}^{\sigma(j-1)}-\left\langle E_{\mathbf{n}^{\prime}}^{\sigma} N_{\mathbf{n}-\mathbf{n}^{\prime}}^{\sigma(j-1)}\right\rangle\right)\right] .
$$

As an example, if the expansion is carried out up to third order, series (51) reads

$$
\begin{aligned}
& N_{\mathbf{n}}^{\sigma}=i\left(\mathbf{g}_{\mathbf{n}}^{\sigma} \cdot \frac{\mathbf{k}}{k}\right) E_{\mathbf{n}} f_{\sigma}+i h_{\mathbf{n}}^{\sigma} E_{\mathbf{n}}^{\sigma} f_{\sigma}-\int d \mathbf{n}_{1} \int d \mathbf{n}_{2} \delta\left(\mathbf{n}-\mathbf{n}_{1}-\mathbf{n}_{2}\right)\left[\left(\mathbf{g}_{\mathbf{n}}^{\sigma} \cdot \frac{\mathbf{k}_{1}}{k_{1}}\right)\left(\mathbf{g}_{\mathbf{n}_{2}}^{\sigma} \cdot \frac{\mathbf{k}_{2}}{k_{2}}\right)\left(E_{\mathbf{n}_{1}} E_{\mathbf{n}_{2}}-\left\langle E_{\mathbf{n}_{1}} E_{\mathbf{n}_{2}}\right\rangle\right)\right. \\
& \left.+\left(\mathbf{g}_{\mathbf{n}}^{\sigma} \cdot \frac{\mathbf{k}_{1}}{k_{1}}\right) h_{\mathbf{n}_{2}}^{\sigma}\left(E_{\mathbf{n}_{1}} E_{\mathbf{n}_{2}}^{\sigma}-\left\langle E_{\mathbf{n}_{1}} E_{\mathbf{n}_{2}}^{\sigma}\right)\right)+h_{\mathbf{n}}^{\sigma}\left(\mathbf{g}_{\mathbf{n}_{2}}^{\sigma} \cdot \frac{\mathbf{k}_{2}}{k_{2}}\right)\left(E_{\mathbf{n}_{1}}^{\sigma} E_{\mathbf{n}_{2}}-\left\langle E_{\mathbf{n}_{1}}^{\sigma} E_{\mathbf{n}_{2}}\right\rangle\right)+h_{\mathbf{n}}^{\sigma} h_{\mathbf{n}_{2}}^{\sigma}\left(E_{\mathbf{n}_{1}}^{\sigma} E_{\mathbf{n}_{2}}^{\sigma}-\left\langle E_{\mathbf{n}_{1}}^{\sigma} E_{\mathbf{n}_{2}}^{\sigma}\right\rangle\right)\right] f_{\sigma} \\
& -i \int d \mathbf{n}_{1} \int d \mathbf{n}_{2} \int d \mathbf{n}_{3} \delta\left(\mathbf{n}-\mathbf{n}_{1}-\mathbf{n}_{2}-\mathbf{n}_{3}\right)\left\{( \mathbf { g } _ { \mathbf { n } } ^ { \sigma } \cdot \frac { \mathbf { k } _ { 3 } } { k _ { 3 } } ) \left[\left(\mathbf{g}_{\mathbf{n}-\mathbf{n}_{3}}^{\sigma} \cdot \frac{\mathbf{k}_{1}}{k_{1}}\right)\left(\mathbf{g}_{\mathbf{n}_{2}}^{\sigma} \cdot \frac{\mathbf{k}_{2}}{k_{2}}\right)\left(E_{\mathbf{n}_{1}} E_{\mathbf{n}_{2}} E_{\mathbf{n}_{3}}-\left\langle E_{\mathbf{n}_{1}} E_{\mathbf{n}_{2}}\right\rangle E_{\mathbf{n}_{3}}-\left\langle E_{\mathbf{n}_{1}} E_{\mathbf{n}_{2}} E_{\mathbf{n}_{3}}\right\rangle\right)\right.\right. \\
& \left.+\left(\mathbf{g}_{\mathbf{n}-\mathbf{n}_{3}}^{\sigma} \cdot \frac{\mathbf{k}_{1}}{k_{1}}\right) h_{\mathbf{n}_{2}}^{\sigma}\left(E_{\mathbf{n}_{1}} E_{\mathbf{n}_{2}}^{\sigma} E_{\mathbf{n}_{3}}-\left\langle E_{\mathbf{n}_{1}} E_{\mathbf{n}_{2}}^{\sigma}\right\rangle E_{\mathbf{n}_{3}}+\left\langle E_{\mathbf{n}_{1}} E_{\mathbf{n}_{2}}^{\sigma} E_{\mathbf{n}_{3}}\right\rangle\right)\right]+h_{\mathbf{n}}^{\sigma}\left[\left(E_{\mathbf{n}_{1}} E_{\mathbf{n}_{2}} E_{\mathbf{n}_{3}}^{\sigma}-\left\langle E_{\mathbf{n}_{1}} E_{\mathbf{n}_{2}}\right\rangle E_{\mathbf{n}_{3}}^{\sigma}-\left\langle E_{\mathbf{n}_{1}} E_{\mathbf{n}_{2}} E_{\mathbf{n}_{3}}^{\sigma}\right\rangle\right)\right. \\
& \left.\times\left(\mathbf{g}_{\mathbf{n}-\mathbf{n}_{3}}^{\sigma} \cdot \frac{\mathbf{k}_{1}}{k_{1}}\right)\left(\mathbf{g}_{\mathbf{n}_{2}}^{\sigma} \cdot \frac{\mathbf{k}_{2}}{k_{2}}\right)+\left(\mathbf{g}_{\mathbf{n}-\mathbf{n}_{3}}^{\sigma} \cdot \frac{\mathbf{k}_{1}}{k_{1}}\right) h_{\mathbf{n}_{2}}^{\sigma}\left(E_{\mathbf{n}_{1}} E_{\mathbf{n}_{2}}^{\sigma} E_{\mathbf{n}_{3}}^{\sigma}-\left\langle E_{\mathbf{n}_{1}} E_{\mathbf{n}_{2}}^{\sigma}\right\rangle E_{\mathbf{n}_{3}}^{\sigma}+\left\langle E_{\mathbf{n}_{1}} E_{\mathbf{n}_{2}}^{\sigma} E_{\mathbf{n}_{3}}^{\sigma}\right\rangle\right)\right] \\
& +\left(\mathbf{g}_{\mathbf{n}}^{\sigma} \cdot \frac{\mathbf{k}_{3}}{k_{3}}\right)\left[h_{\mathbf{n}-\mathbf{n}_{3}}^{\sigma}\left(\mathbf{g}_{\mathbf{n}_{2}}^{\sigma} \cdot \frac{\mathbf{k}_{2}}{k_{2}}\right)\left(E_{\mathbf{n}_{1}}^{\sigma} E_{\mathbf{n}_{2}} E_{\mathbf{n}_{3}}-\left\langle E_{\mathbf{n}_{1}}^{\sigma} E_{\mathbf{n}_{2}}\right\rangle E_{\mathbf{n}_{3}}-\left\langle E_{\mathbf{n}_{1}}^{\sigma} E_{\mathbf{n}_{2}} E_{\mathbf{n}_{3}}\right)\right)+h_{\mathbf{n}-\mathbf{n}_{3}}^{\sigma} h_{\mathbf{n}_{2}}^{\sigma}\left(E_{\mathbf{n}_{1}}^{\sigma} E_{\mathbf{n}_{2}}^{\sigma} E_{\mathbf{n}_{3}}-\left\langle E_{\mathbf{n}_{1}}^{\sigma} E_{\mathbf{n}_{2}}^{\sigma}\right\rangle E_{\mathbf{n}_{3}}\right.\right. \\
& \left.\left.-\left\langle E_{\mathbf{n}_{1}}^{\sigma} E_{\mathbf{n}_{2}}^{\sigma} E_{\mathbf{n}_{3}}\right\rangle\right)\right]+h_{\mathbf{n}}^{\sigma}\left[h_{\mathbf{n}-\mathbf{n}_{3}}^{\sigma}\left(\mathbf{g}_{\mathbf{n}_{2}}^{\sigma} \cdot \frac{\mathbf{k}_{2}}{k_{2}}\right)\left(E_{\mathbf{n}_{1}}^{\sigma} E_{\mathbf{n}_{2}} E_{\mathbf{n}_{3}}^{\sigma}-\left\langle E_{\mathbf{n}_{1}}^{\sigma} E_{\mathbf{n}_{2}}\right\rangle E_{\mathbf{n}_{3}}^{\sigma}-\left\langle E_{\mathbf{n}_{1}}^{\sigma} E_{\mathbf{n}_{2}} E_{\mathbf{n}_{3}}^{\sigma}\right\rangle\right)\right. \\
& \left.\left.+h_{\mathbf{n}-\mathbf{n}_{3}}^{\sigma} h_{\mathbf{n}_{2}}^{\sigma}\left(E_{\mathbf{n}_{1}}^{\sigma} E_{\mathbf{n}_{2}}^{\sigma} E_{\mathbf{n}_{3}}^{\sigma}-\left\langle E_{\mathbf{n}_{1}}^{\sigma} E_{\mathbf{n}_{2}}^{\sigma}\right\rangle E_{\mathbf{n}_{3}}^{\sigma}-\left\langle E_{\mathbf{n}_{1}}^{\sigma} E_{\mathbf{n}_{2}}^{\sigma} E_{\mathbf{n}_{3}}^{\sigma}\right\rangle\right)\right]\right\} f_{\sigma} .
\end{aligned}
$$

Such an expansion would be sufficient to take into account the interactions between the waves and the nonlinear wave-particle interactions [31]. For the purpose of the present paper it is sufficient to carry out expansion (51) up to first order, in the form of (52), to take into account the quasilinear wave-particle interactions [31].

The expansion of Eq. (47) develops in the same way,

$$
N_{\mathbf{n}}^{d}=\sum_{j=1}^{\infty} N_{\mathbf{n}}^{d(j)}
$$

with the first and the $j$ th-order terms given respectively by

$$
\begin{gathered}
N_{\mathbf{n}}^{d(1)}=i h_{\mathbf{n}}^{d} \hat{E}_{\mathbf{n}} f_{d}+i\left(\mathbf{g}_{\mathbf{n}}^{d} \cdot \frac{\mathbf{k}}{k}\right) E_{\mathbf{n}} f_{d}, \\
N_{\mathbf{n}}^{d(j)}=i \int d \mathbf{n}^{\prime}\left[h_{\mathbf{n}}^{d}\left(\hat{E}_{\mathbf{n}^{\prime}} N_{\mathbf{n}-\mathbf{n}^{\prime}}^{d(j-1)}-\left\langle\hat{E}_{\mathbf{n}^{\prime}} N_{\mathbf{n}-\mathbf{n}^{\prime}}^{d(j-1)}\right\rangle\right)+\mathbf{g}_{\mathbf{n}}^{d}\left(N_{\mathbf{n}-\mathbf{n}^{\prime}}^{d(j-1)} \cdot \frac{\mathbf{k}^{\prime}}{k^{\prime}} E_{\mathbf{n}^{\prime}}-\left\langle N_{\mathbf{n}-\mathbf{n}^{\prime}}^{d(j-1)} \cdot \frac{\mathbf{k}^{\prime}}{k^{\prime}} E_{\mathbf{n}^{\prime}}\right\rangle\right)\right],
\end{gathered}
$$

and has a similar expanded form as (54), given by

$$
\begin{aligned}
N_{\mathbf{n}}^{d}= & i h_{\mathbf{n}}^{d} \hat{E}_{\mathbf{n}} f_{d}+i\left(\mathbf{g}_{\mathbf{n}}^{d} \cdot \frac{\mathbf{k}}{k}\right) E_{\mathbf{n}} f_{d}-\int d \mathbf{n}_{1} \int d \mathbf{n}_{2} \delta\left(\mathbf{n}-\mathbf{n}_{1}-\mathbf{n}_{2}\right)\left[h_{\mathbf{n}}^{d} h_{\mathbf{n}_{2}}^{d}\left(\hat{E}_{\mathbf{n}_{1}} \hat{E}_{\mathbf{n}_{2}}-\left\langle\hat{E}_{\mathbf{n}_{1}} \hat{E}_{\mathbf{n}_{2}}\right\rangle\right)+\left(\mathbf{g}_{\mathbf{n}}^{d} \cdot \frac{\mathbf{k}_{1}}{k_{1}}\right) h_{\mathbf{n}_{2}}^{d}\left(\hat{E}_{\mathbf{n}_{2}} E_{\mathbf{n}_{1}}-\left\langle\hat{E}_{\mathbf{n}_{2}} E_{\mathbf{n}_{1}}\right\rangle\right)\right. \\
& \left.+\left(\hat{E}_{\mathbf{n}_{1}} E_{\mathbf{n}_{2}}-\left\langle\hat{E}_{\mathbf{n}_{1}} E_{\mathbf{n}_{2}}\right\rangle\right) h_{\mathbf{n}}^{d}\left(\mathbf{g}_{\mathbf{n}_{2}}^{d} \cdot \frac{\mathbf{k}_{2}}{k_{2}}\right)+\left(\mathbf{g}_{\mathbf{n}}^{d} \cdot \frac{\mathbf{k}_{1}}{k_{1}}\right)\left(\mathbf{g}_{\mathbf{n}_{2}}^{d} \cdot \frac{\mathbf{k}_{2}}{k_{2}}\right)\left(E_{\mathbf{n}_{2}} E_{\mathbf{n}_{1}}-\left\langle E_{\mathbf{n}_{2}} E_{\mathbf{n}_{1}}\right\rangle\right)\right] f_{d} \\
& -i \int d \mathbf{n}_{1} \int d \mathbf{n}_{2} \int d \mathbf{n}_{3} \delta\left(\mathbf{n}-\mathbf{n}_{1}-\mathbf{n}_{2}-\mathbf{n}_{3}\right)\left\{h _ { \mathbf { n } } ^ { d } \left[h_{\mathbf{n}-\mathbf{n}_{3}}^{d} h_{\mathbf{n}_{2}}^{d}\left(\hat{E}_{\mathbf{n}_{1}} \hat{E}_{\mathbf{n}_{2}} \hat{E}_{\mathbf{n}_{3}}-\left\langle\hat{E}_{\mathbf{n}_{1}} \hat{E}_{\mathbf{n}_{2}}\right\rangle \hat{E}_{\mathbf{n}_{3}}-\left\langle\hat{E}_{\mathbf{n}_{1}} \hat{E}_{\mathbf{n}_{2}} \hat{E}_{\mathbf{n}_{3}}\right\rangle\right)\right.\right. \\
& \left.+\left(\mathbf{g}_{\mathbf{n}-\mathbf{n}_{3}}^{d} \cdot \frac{\mathbf{k}_{1}}{k_{1}}\right) h_{\mathbf{n}_{2}}^{d}\left(E_{\mathbf{n}_{1}} \hat{E}_{\mathbf{n}_{2}} \hat{E}_{\mathbf{n}_{3}}-\left\langle E_{\mathbf{n}_{1}} \hat{E}_{\mathbf{n}_{2}}\right\rangle \hat{E}_{\mathbf{n}_{3}}-\left\langle E_{\mathbf{n}_{1}} \hat{E}_{\mathbf{n}_{2}} \hat{E}_{\mathbf{n}_{3}}\right\rangle\right)\right] \\
& +\left(\mathbf{g}_{\mathbf{n}}^{d} \cdot \frac{\mathbf{k}_{3}}{k_{3}}\right)\left[h_{\mathbf{n}-\mathbf{n}_{3}}^{d} h_{\mathbf{n}_{2}}^{d}\left(\hat{E}_{\mathbf{n}_{1}} \hat{E}_{\mathbf{n}_{2}} E_{\mathbf{n}_{3}}-\left\langle\hat{E}_{\mathbf{n}_{1}} \hat{E}_{\mathbf{n}_{2}}\right\rangle E_{\mathbf{n}_{3}}-\left\langle\hat{E}_{\mathbf{n}_{1}} \hat{E}_{\mathbf{n}_{2}} E_{\mathbf{n}_{3}}\right\rangle\right)\right. \\
& \left.+\left(\mathbf{g}_{\mathbf{n}-\mathbf{n}_{3}}^{d} \cdot \frac{\mathbf{k}_{1}}{k_{1}}\right) h_{\mathbf{n}_{2}}^{d}\left(E_{\mathbf{n}_{1}} \hat{E}_{\mathbf{n}_{2}} E_{\mathbf{n}_{3}}-\left\langle E_{\mathbf{n}_{1}} \hat{E}_{\mathbf{n}_{2}}\right\rangle E_{\mathbf{n}_{3}}-\left\langle E_{\mathbf{n}_{1}} \hat{E}_{\mathbf{n}_{2}} E_{\mathbf{n}_{3}}\right\rangle\right)\right]
\end{aligned}
$$

023102-6 


$$
\begin{aligned}
& +\left[\left(\hat{E}_{\mathbf{n}_{1}} E_{\mathbf{n}_{2}} \hat{E}_{\mathbf{n}_{3}}-\left\langle\hat{E}_{\mathbf{n}_{1}} E_{\mathbf{n}_{2}}\right\rangle \hat{E}_{\mathbf{n}_{3}}-\left\langle\hat{E}_{\mathbf{n}_{1}} E_{\mathbf{n}_{2}} \hat{E}_{\mathbf{n}_{3}}\right\rangle\right) h_{\mathbf{n}}^{d} h_{\mathbf{n}-\mathbf{n}_{3}}^{d}+\left(E_{\mathbf{n}_{1}} E_{\mathbf{n}_{2}} \hat{E}_{\mathbf{n}_{3}}-\left\langle E_{\mathbf{n}_{1}} E_{\mathbf{n}_{2}}\right\rangle \hat{E}_{\mathbf{n}_{3}}-\left\langle E_{\mathbf{n}_{1}} E_{\mathbf{n}_{2}} \hat{E}_{\mathbf{n}_{3}}\right\rangle\right) h_{\mathbf{n}}^{d}\left(\mathbf{g}_{\mathbf{n}-\mathbf{n}_{3}}^{d} \cdot \frac{\mathbf{k}_{1}}{k_{1}}\right)\right. \\
& +\left(\hat{E}_{\mathbf{n}_{1}} E_{\mathbf{n}_{2}} E_{\mathbf{n}_{3}}-\left\langle\hat{E}_{\mathbf{n}_{1}} E_{\mathbf{n}_{2}}\right\rangle E_{\mathbf{n}_{3}}-\left\langle\hat{E}_{\mathbf{n}_{1}} E_{\mathbf{n}_{2}} E_{\mathbf{n}_{3}}\right\rangle\right)\left(\mathbf{g}_{\mathbf{n}}^{d} \cdot \frac{\mathbf{k}_{3}}{k_{3}}\right) h_{\mathbf{n}-\mathbf{n}_{3}}^{d} \\
& \left.\left.+\left(E_{\mathbf{n}_{1}} E_{\mathbf{n}_{2}} E_{\mathbf{n}_{3}}-\left\langle E_{\mathbf{n}_{1}} E_{\mathbf{n}_{2}}\right\rangle E_{\mathbf{n}_{3}}-\left\langle E_{\mathbf{n}_{1}} E_{\mathbf{n}_{2}} E_{\mathbf{n}_{3}}\right\rangle\right)\left(\mathbf{g}_{\mathbf{n}}^{d} \cdot \frac{\mathbf{k}_{3}}{k_{3}}\right)\left(\mathbf{g}_{\mathbf{n}-\mathbf{n}_{3}}^{d} \cdot \frac{\mathbf{k}_{1}}{k_{1}}\right)\right]\left(\mathbf{g}_{\mathbf{n}_{2}}^{d} \cdot \frac{\mathbf{k}_{2}}{k_{2}}\right)\right\} f_{d} .
\end{aligned}
$$

The set of equations which governs the time evolution of the dust-plasma system, in the quasilinear approximation, is therefore given by Eqs. (52) and (56), which are respectively limit cases of (54) and (58), for the fluctuations in particle and dust populations, and Eqs. (34) and (35) for the average distributions, with the fluctuating fields given by Eqs. (41), (42), and (50).

\section{QUASILINEAR APPROXIMATION}

In neglect of nonlinear effects, only terms proportional to $f_{\sigma}$ and $\omega_{\sigma}$ will remain at the right-hand side of Eq. (19), and terms proportional to $I$ and to $\partial f_{d} / \partial q$ will remain at the right-hand side of Eq. (20).

A coupling of the different electric field sources explicitly arises by insertion of Eqs. (52) and (56) into (41), (42), and (50). Multiplying the resulting equations by $E_{-\mathbf{n}}$ from the right and taking the average, we obtain

$$
\begin{gathered}
\left\langle E_{\mathbf{n}}^{2}\right\rangle(\epsilon+\psi)=\sum_{\sigma} \chi_{1 \sigma}\left\langle E_{\mathbf{n}}^{\sigma} E_{-\mathbf{n}}\right\rangle+\psi_{1}\left\langle\hat{E}_{\mathbf{n}} E_{-\mathbf{n}}\right\rangle \\
\left\langle E_{\mathbf{n}}^{\sigma} E_{-\mathbf{n}}\right\rangle=\zeta_{1 \sigma}\left\langle\hat{E}_{\mathbf{n}} E_{-\mathbf{n}}\right\rangle+\zeta_{2 \sigma}\left\langle E_{\mathbf{n}}^{2}\right\rangle \\
\left\langle\hat{E}_{\mathbf{n}} E_{-\mathbf{n}}\right\rangle=\rho_{1}\left\langle E_{\mathbf{n}}^{2}\right\rangle+\sum_{\sigma} \rho_{2 \sigma}\left\langle E_{\mathbf{n}}^{\sigma} E_{-\mathbf{n}}\right\rangle
\end{gathered}
$$

Here some response functions can be identified. The first one is the well-known expression of the dielectric function,

$$
\epsilon(\mathbf{n})=1+\chi(\mathbf{n}),
$$

where $\chi(\mathbf{n})$ is the linear electric susceptibility,

$$
\chi(\mathbf{n})=-\frac{4 \pi}{k^{2}} \sum_{\sigma} q_{\sigma} \int d \mathbf{v}\left(\mathbf{g}_{\mathbf{n}}^{\sigma} \cdot \mathbf{k}\right) f_{\sigma} .
$$

Expression (63) together with (43) are known modified susceptibilities expressions that can be found in kinetic formulations with a nonzero collisional term describing the inelastic collision absorption [32,33]. The other ones are due to the presence of dust and contribute to the effective linear susceptibility,

$$
\begin{gathered}
\psi(\mathbf{n})=-\frac{4 \pi}{k^{2}} \int d q q \int d \mathbf{v}\left(\mathbf{g}_{\mathbf{n}}^{d} \cdot \mathbf{k}\right) f_{d}, \\
\chi_{1 \sigma}(\mathbf{n})=\frac{4 \pi}{k^{2}} q_{\sigma} \int d \mathbf{v} h_{\mathbf{n}}^{\sigma} k f_{\sigma}, \\
\psi_{1}(\mathbf{n})=\frac{4 \pi}{k^{2}} \int d q q \int d \mathbf{v} h_{\mathbf{n}}^{d} k f_{d}, \\
\rho_{1}(\mathbf{n})=\frac{4 \pi}{k^{2}} \sum_{\sigma} \frac{q_{\sigma}^{3}}{e^{2}} \frac{m_{d}}{m_{\sigma}} \frac{1}{n_{d}} \int d \mathbf{v} d q f_{d} \\
\times \int d \mathbf{v}^{\prime} \Omega_{\mathbf{k}}^{\sigma}\left(\mathbf{v}-\mathbf{v}^{\prime}, a\right)\left(\mathbf{g}_{\mathbf{n}}^{\sigma} \cdot \mathbf{k}\right) f_{\sigma}, \\
\rho_{2 \sigma}(\mathbf{n})=\frac{4 \pi}{k^{2}} \frac{q_{\sigma}^{3}}{e^{2}} \frac{m_{d}}{m_{\sigma}} \frac{1}{n_{d}} \int d \mathbf{v} d q f_{d} \\
\times \int d \mathbf{v}^{\prime} \Omega_{\mathbf{k}}^{\sigma}\left(\mathbf{v}-\mathbf{v}^{\prime}, a\right) h_{\mathbf{n}}^{\sigma} k f_{\sigma},
\end{gathered}
$$

$$
\begin{gathered}
\zeta_{1 \sigma}(\mathbf{n})=\frac{4 \pi}{k^{2}} \frac{q_{\sigma}}{n_{\sigma}} \int d \mathbf{v} f_{\sigma} \int d \mathbf{v}^{\prime} d q^{\prime} \Omega_{\mathbf{k}}^{\sigma}\left(\mathbf{v}-\mathbf{v}^{\prime}, a\right) h_{\mathbf{n}}^{d} k f_{d}, \\
\zeta_{2 \sigma}(\mathbf{n})=\frac{4 \pi}{k^{2}} \frac{q_{\sigma}}{n_{\sigma}} \int d \mathbf{v} f_{\sigma} \int d \mathbf{v}^{\prime} d q^{\prime} \Omega_{\mathbf{k}}^{\sigma}\left(\mathbf{v}-\mathbf{v}^{\prime}, a\right)\left(\mathbf{g}_{\mathbf{n}}^{d} \cdot \mathbf{k}\right) f_{d} .
\end{gathered}
$$

Substitution of (60) in Eqs. (59) and (61), and further substitution of the succeeding expression in Eq. (59), leads to

$$
\begin{aligned}
\left\langle E_{\mathbf{n}}^{2}\right\rangle\left[\epsilon+\psi-\sum_{\sigma} \chi_{1 \sigma}\left(\zeta_{1 \sigma} \frac{\rho_{1}+\sum_{\sigma^{\prime}} \rho_{2 \sigma^{\prime}} \zeta_{2 \sigma^{\prime}}}{1-\sum_{\sigma^{\prime}} \rho_{2 \sigma^{\prime}} \zeta_{1 \sigma^{\prime}}}+\zeta_{2 \sigma}\right)\right. \\
\left.-\psi_{1} \frac{\rho_{1}+\sum_{\sigma^{\prime}} \rho_{2 \sigma^{\prime}} \zeta_{2 \sigma^{\prime}}}{1-\sum_{\sigma^{\prime}} \rho_{2 \sigma^{\prime}} \zeta_{1 \sigma^{\prime}}}\right]=0
\end{aligned}
$$

Defining by $D_{1}$ the quantity that multiplies the spectral density, the identity

$$
\begin{aligned}
D_{1}= & \epsilon+\psi-\sum_{\sigma} \chi_{1 \sigma}\left(\zeta_{1 \sigma} \frac{\rho_{1}+\sum_{\sigma^{\prime}} \rho_{2 \sigma^{\prime}} \zeta_{2 \sigma^{\prime}}}{1-\sum_{\sigma^{\prime}} \rho_{2 \sigma^{\prime}} \zeta_{1 \sigma^{\prime}}}+\zeta_{2 \sigma}\right) \\
& -\psi_{1} \frac{\rho_{1}+\sum_{\sigma^{\prime}} \rho_{2 \sigma^{\prime}} \zeta_{2 \sigma^{\prime}}}{1-\sum_{\sigma^{\prime}} \rho_{2 \sigma^{\prime}} \zeta_{1 \sigma^{\prime}}}=0
\end{aligned}
$$

is the dispersion relation related to the spectral energy $\left\langle E_{\mathbf{n}}^{2}\right\rangle$.

Denoting by $\omega_{1 \mathbf{k}}^{\alpha}$ the angular frequencies which are the roots of the dispersion relation (72), we assume that the only field fluctuations to be present are those associated to these frequencies. Moreover, assuming a random-phase approximation, we will have

$$
\left\langle E_{\mathbf{n}}^{2}\right\rangle=\sum_{\alpha} \sum_{j= \pm} \mathcal{I}_{\mathbf{k}}^{\alpha j} \delta\left(\omega+j \omega_{1 \mathbf{k}}^{\alpha}\right),
$$

where $\mathcal{I}_{\mathbf{k}}^{\alpha j}$ denotes the total intensity of the fluctuations.

We expand the dielectric linear functions under the assumption that $|\operatorname{Im}(\omega)| \ll|\omega|$, and take into account that the imaginary part of the frequency incorporates the slow variation of the wave amplitudes. Therefore [13],

$$
\begin{aligned}
& {[\epsilon(\mathbf{n})+\psi(\mathbf{n})]\left\langle E_{\mathbf{n}}^{2}\right\rangle} \\
& \quad \simeq\left[\epsilon(\mathbf{n})+\psi(\mathbf{n})+\frac{i}{2}\left(\frac{\partial \operatorname{Re} \epsilon}{\partial \omega}+\frac{\partial \operatorname{Re} \psi}{\partial \omega}\right) \frac{\partial}{\partial t}\right]\left\langle E_{\mathbf{n}}^{2}\right\rangle .
\end{aligned}
$$


From Eq. (71) we obtain

$$
\frac{i}{2}\left(\frac{\partial \operatorname{Re} \epsilon}{\partial \omega}+\frac{\partial \operatorname{Re} \psi}{\partial \omega}\right) \frac{\partial}{\partial t}\left\langle E_{\mathbf{n}}^{2}\right\rangle=-(\epsilon(\mathbf{n})+\psi(\mathbf{n}))\left\langle E_{\mathbf{n}}^{2}\right\rangle+\left\langle E_{\mathbf{n}}^{2}\right\rangle\left[\sum_{\sigma} \chi_{1 \sigma}\left(\zeta_{1 \sigma} \frac{\rho_{1}+\sum_{\sigma^{\prime}} \rho_{2 \sigma^{\prime}} \zeta_{2 \sigma^{\prime}}}{1-\sum_{\sigma^{\prime}} \rho_{2 \sigma^{\prime}} \zeta_{1 \sigma^{\prime}}}+\zeta_{2 \sigma}\right)+\psi_{1} \frac{\rho_{1}+\sum_{\sigma^{\prime}} \rho_{2 \sigma^{\prime}} \zeta_{2 \sigma^{\prime}}}{1-\sum_{\sigma^{\prime}} \rho_{2 \sigma^{\prime}} \zeta_{1 \sigma^{\prime}}}\right]
$$

The time evolution of $\left\langle E_{\mathbf{n}}^{2}\right\rangle$ can be obtained by equating the imaginary part of (75) to zero,

$$
\begin{aligned}
\frac{1}{2}\left(\frac{\partial \operatorname{Re} \epsilon}{\partial \omega}+\frac{\partial \operatorname{Re} \psi}{\partial \omega}\right) \frac{\partial}{\partial t}\left\langle E_{\mathbf{n}}^{2}\right\rangle= & -\operatorname{Im}[\epsilon(\mathbf{n})+\psi(\mathbf{n})]\left\langle E_{\mathbf{n}}^{2}\right\rangle \\
& +\operatorname{Im}\left[\sum_{\sigma} \chi_{1 \sigma}\left(\zeta_{1 \sigma} \frac{\rho_{1}+\sum_{\sigma^{\prime}} \rho_{2 \sigma^{\prime}} \zeta_{2 \sigma^{\prime}}}{1-\sum_{\sigma^{\prime}} \rho_{2 \sigma^{\prime}} \zeta_{1 \sigma^{\prime}}}+\zeta_{2 \sigma}\right)+\psi_{1} \frac{\rho_{1}+\sum_{\sigma^{\prime}} \rho_{2 \sigma^{\prime}} \zeta_{2 \sigma^{\prime}}}{1-\sum_{\sigma^{\prime}} \rho_{2 \sigma^{\prime}} \zeta_{1 \sigma^{\prime}}}\right]\left\langle E_{\mathbf{n}}^{2}\right\rangle
\end{aligned}
$$

Inserting (73) into (76) and integrating in $\omega$, we find the kinetic equation for the intensity of the waves,

$$
\begin{aligned}
\frac{\operatorname{Re}}{2}\left[\frac{\partial \epsilon\left(j \omega_{1 \mathbf{k}}^{\alpha}\right)}{\partial \omega}+\frac{\partial \psi\left(j \omega_{1 \mathbf{k}}^{\alpha}\right)}{\partial \omega}\right] \frac{\partial \mathcal{I}_{\mathbf{k}}^{\alpha j}}{\partial t}= & -\operatorname{Im}\left(\epsilon\left(j \omega_{1 \mathbf{k}}^{\alpha}\right)+\psi\left(j \omega_{1 \mathbf{k}}^{\alpha}\right)\right) \mathcal{I}_{\mathbf{k}}^{\alpha j}+\operatorname{Im}\left[\sum_{\sigma} \chi_{1 \sigma}\left(\zeta_{1 \sigma} \frac{\rho_{1}+\sum_{\sigma^{\prime}} \rho_{2 \sigma^{\prime}} \zeta_{2 \sigma^{\prime}}}{1-\sum_{\sigma^{\prime}} \rho_{2 \sigma^{\prime}} \zeta_{1 \sigma^{\prime}}}+\zeta_{2 \sigma}\right)\right. \\
& \left.+\psi_{1} \frac{\rho_{1}+\sum_{\sigma^{\prime}} \rho_{2 \sigma^{\prime}} \zeta_{2 \sigma^{\prime}}}{1-\sum_{\sigma^{\prime}} \rho_{2 \sigma^{\prime}} \zeta_{1 \sigma^{\prime}}}\right] \mathcal{I}_{\mathbf{k}}^{\alpha j}
\end{aligned}
$$

For $\left\langle\hat{E}_{\mathbf{n}}^{2}\right\rangle$ we proceed with a similar approach but multiplying Eqs. (41), (42), and (50) by $\hat{E}_{-\mathbf{n}}$. Another set of three equations is obtained in the form

$$
\begin{gathered}
\left\langle E_{\mathbf{n}} \hat{E}_{-\mathbf{n}}\right\rangle(\epsilon+\psi)=\sum_{\sigma} \chi_{1 \sigma}\left\langle E_{\mathbf{n}}^{\sigma} \hat{E}_{-\mathbf{n}}\right\rangle+\psi_{1}\left\langle\hat{E}_{\mathbf{n}}^{2}\right\rangle, \\
\left\langle E_{\mathbf{n}}^{\sigma} \hat{E}_{-\mathbf{n}}\right\rangle=\zeta_{1 \sigma}\left\langle\hat{E}_{\mathbf{n}}^{2}\right\rangle+\zeta_{2 \sigma}\left\langle E_{\mathbf{n}} \hat{E}_{-\mathbf{n}}\right\rangle, \\
\left\langle\hat{E}_{\mathbf{n}}^{2}\right\rangle=\rho_{1}\left\langle E_{\mathbf{n}} \hat{E}_{-\mathbf{n}}\right\rangle+\sum_{\sigma} \rho_{2 \sigma}\left\langle E_{\mathbf{n}}^{\sigma} \hat{E}_{-\mathbf{n}}\right\rangle .
\end{gathered}
$$

By inserting Eq. (79) in Eqs. (78) and (80), and combining the succeeding expressions, we obtain

$$
\left\langle\hat{E}_{\mathbf{n}}^{2}\right\rangle\left[1-\rho_{1} \frac{\sum_{\sigma} \chi_{1 \sigma} \zeta_{1 \sigma}+\psi_{1}}{\epsilon+\psi-\sum_{\sigma} \chi_{1 \sigma} \zeta_{2 \sigma}}-\sum_{\sigma} \rho_{2 \sigma}\left(\zeta_{1 \sigma}+\zeta_{2 \sigma} \frac{\sum_{\sigma^{\prime}} \chi_{1 \sigma^{\prime}} \zeta_{1 \sigma^{\prime}}+\psi_{1}}{\epsilon+\psi-\sum_{\sigma^{\prime}} \chi_{1 \sigma^{\prime}} \zeta_{2 \sigma^{\prime}}}\right)\right]=0,
$$

which leads to the dispersion relation associated to $\left\langle\hat{E}_{\mathbf{n}}^{2}\right\rangle$,

$$
D_{2}=1-\rho_{1} \frac{\sum_{\sigma} \chi_{1 \sigma} \zeta_{1 \sigma}+\psi_{1}}{\epsilon+\psi-\sum_{\sigma} \chi_{1 \sigma} \zeta_{2 \sigma}}-\sum_{\sigma} \rho_{2 \sigma}\left(\zeta_{1 \sigma}+\zeta_{2 \sigma} \frac{\sum_{\sigma^{\prime}} \chi_{1 \sigma^{\prime}} \zeta_{1 \sigma^{\prime}}+\psi_{1}}{\epsilon+\psi-\sum_{\sigma^{\prime}} \chi_{1 \sigma^{\prime}} \zeta_{2 \sigma^{\prime}}}\right)=0
$$

By analogy with $\left\langle E_{\mathbf{n}}^{2}\right\rangle$, we assume

$$
\left\langle\hat{E}_{\mathbf{n}}^{2}\right\rangle=\sum_{\alpha} \sum_{j= \pm} \hat{\mathcal{I}}_{\mathbf{k}}^{\alpha j} \delta\left(\omega+j \omega_{2 \mathbf{k}}^{\alpha}\right),
$$

where $\omega_{2 \mathbf{k}}^{\alpha}$ are the roots of $D_{2}$, with the time evolution equation for the intensity of the fluctuations given by

$$
\frac{\operatorname{Re}}{2}\left[\frac{\partial D_{2}\left(j \omega_{2 \mathbf{k}}^{\alpha}\right)}{\partial \omega}\right] \frac{\partial \hat{\mathcal{I}}_{\mathbf{k}}^{\alpha j}}{\partial t}=-\operatorname{Im}\left[D_{2}\left(j \omega_{2 \mathbf{k}}^{\alpha}\right)\right] \hat{\mathcal{I}}_{\mathbf{k}}^{\alpha j} .
$$

For $\left\langle E_{\mathbf{n}}^{\sigma^{\prime}} E_{-\mathbf{n}}^{\sigma}\right\rangle$, considering the same equations (41), (42), and (50), but multiplied by $E_{-\mathbf{n}}^{\sigma}$,

$$
\begin{aligned}
&\left\langle E_{\mathbf{n}} E_{-\mathbf{n}}^{\sigma}\right\rangle(\epsilon+\psi)=\sum_{\sigma^{\prime \prime}} \chi_{1 \sigma^{\prime \prime}}\left\langle E_{\mathbf{n}}^{\sigma^{\prime \prime}} E_{-\mathbf{n}}^{\sigma}\right\rangle+\psi_{1}\left\langle\hat{E}_{\mathbf{n}} E_{-\mathbf{n}}^{\sigma}\right\rangle, \\
&\left\langle E_{\mathbf{n}}^{\sigma^{\prime}} E_{-\mathbf{n}}^{\sigma}\right\rangle=\zeta_{1 \sigma^{\prime}}\left\langle\hat{E}_{\mathbf{n}} E_{-\mathbf{n}}^{\sigma}\right\rangle+\zeta_{2 \sigma^{\prime}}\left\langle E_{\mathbf{n}} E_{-\mathbf{n}}^{\sigma}\right\rangle, \\
&\left\langle\hat{E}_{\mathbf{n}} E_{-\mathbf{n}}^{\sigma}\right\rangle=\rho_{1}\left\langle E_{\mathbf{n}} E_{-\mathbf{n}}^{\sigma}\right\rangle+\sum_{\sigma^{\prime \prime}} \rho_{2 \sigma^{\prime \prime}}\left\langle E_{\mathbf{n}}^{\sigma^{\prime \prime}} E_{-\mathbf{n}}^{\sigma}\right\rangle .
\end{aligned}
$$

By combining these equations,

$$
\begin{aligned}
\left\langle E_{\mathbf{n}}^{\sigma^{\prime}} E_{-\mathbf{n}}^{\sigma}\right\rangle= & \zeta_{1 \sigma^{\prime}} \sum_{\sigma^{\prime \prime}} \frac{\epsilon+\psi}{\epsilon+\psi-\rho_{1} \psi_{1}}\left(\frac{\rho_{1} \chi_{1 \sigma^{\prime \prime}}}{\epsilon+\psi}+\rho_{2 \sigma^{\prime \prime}}\right)\left\langle E_{\mathbf{n}}^{\sigma^{\prime \prime}} E_{-\mathbf{n}}^{\sigma}\right\rangle \\
& +\frac{\zeta_{2 \sigma^{\prime}}}{\epsilon+\psi} \sum_{\sigma^{\prime \prime}}\left[\chi_{1 \sigma^{\prime \prime}}\left\langle E_{\mathbf{n}}^{\sigma^{\prime \prime}} E_{-\mathbf{n}}^{\sigma}\right\rangle+\psi_{1} \frac{\epsilon+\psi}{\epsilon+\psi-\rho_{1} \psi_{1}}\left(\frac{\rho_{1} \chi_{1 \sigma^{\prime \prime}}}{\epsilon+\psi}+\rho_{2 \sigma^{\prime \prime}}\right)\left\langle E_{\mathbf{n}}^{\sigma^{\prime \prime}} E_{-\mathbf{n}}^{\sigma}\right\rangle\right] .
\end{aligned}
$$


A dispersion relation

$$
D_{3}=0
$$

can be obtained from the system of equations that arises from (88) when the plasma species, electrons and ions, are defined. An example is given in the following section. If $\omega_{3 \mathbf{k}}^{\alpha}$ are the roots of $D_{3}$, then we may write for each pair $\sigma^{\prime} \sigma$

$$
\frac{\operatorname{Re}}{2}\left[\frac{\partial D_{3}\left(j \omega_{3 \mathbf{k}}^{\alpha}\right)}{\partial \omega}\right] \frac{\partial \mathcal{I}_{\mathbf{k}, \sigma^{\prime} \sigma}^{\alpha j}}{\partial t}=-\operatorname{Im}\left[D_{3}\left(j \omega_{3 \mathbf{k}}^{\alpha}\right)\right] \mathcal{I}_{\mathbf{k}, \sigma^{\prime} \sigma}^{\alpha j},
$$

where, in analogy with the previous two cases,

$$
\left\langle E_{\mathbf{n}}^{\sigma^{\prime}} E_{-\mathbf{n}}^{\sigma}\right\rangle=\sum_{\alpha} \sum_{j= \pm} \mathcal{I}_{\mathbf{k}, \sigma^{\prime} \sigma}^{\alpha j} \delta\left(\omega+j \omega_{3 \mathbf{k}}^{\alpha}\right) .
$$

It is worthwhile to notice that there are as many equations (90) as there are combinations among equal and different plasma species.

The time evolution of the distribution functions of the plasma particles and dust is given by (34) and (35). By substitution of (52) and (56),

$$
\begin{gathered}
\frac{\partial f_{\sigma}\left(\mathbf{v}, t^{\prime}\right)}{\partial t^{\prime}}=-\omega_{\sigma} f_{\sigma}-i \frac{q_{\sigma}}{m_{\sigma}} \int d \mathbf{n} \frac{\mathbf{k}}{k} \cdot \frac{\partial}{\partial \mathbf{v}}\left(\mathbf{g}_{\mathbf{n}}^{\sigma} \cdot \frac{\mathbf{k}}{k}\left\langle E_{\mathbf{n}}^{2}\right\rangle+h_{\mathbf{n}}^{\sigma}\left\langle E_{\mathbf{n}}^{\sigma} E_{-\mathbf{n}}\right\rangle\right) f_{\sigma}-i \frac{q_{\sigma}}{m_{\sigma} v_{*}} \int d \mathbf{n}\left(\mathbf{g}_{\mathbf{n}}^{\sigma} \cdot \frac{\mathbf{k}}{k}\left\langle E_{\mathbf{n}} E_{-\mathbf{n}}^{\sigma}\right\rangle+h_{\mathbf{n}}^{\sigma}\left\langle E_{\mathbf{n}}^{\sigma 2}\right\rangle\right) f_{\sigma},(92) \\
\frac{\partial f_{d}\left(\mathbf{v}, q, t^{\prime}\right)}{\partial t^{\prime}}=-I \frac{\partial f_{d}}{\partial q}-i \frac{q}{m_{d}} \int d \mathbf{n} \frac{\mathbf{k}}{k} \cdot \frac{\partial}{\partial \mathbf{v}}\left(h_{\mathbf{n}}^{d}\left\langle\hat{E}_{\mathbf{n}} E_{-\mathbf{n}}\right\rangle+\mathbf{g}_{\mathbf{n}}^{d} \cdot \frac{\mathbf{k}}{k}\left\langle E_{\mathbf{n}}^{2}\right\rangle\right) f_{d}-i \frac{e^{2}}{m_{d} v_{*}} \frac{\partial}{\partial q} \int d \mathbf{n}\left(h_{\mathbf{n}}^{d}\left\langle\hat{E}_{\mathbf{n}}^{2}\right\rangle+\mathbf{g}_{\mathbf{n}}^{d} \cdot \frac{\mathbf{k}}{k}\left\langle E_{\mathbf{n}} \hat{E}_{-\mathbf{n}}\right\rangle\right) f_{d} .
\end{gathered}
$$

Up to this point only correlations of equal fields were given. The expressions for the correlations with mixed field fluctuations can be obtained from a combination of Eqs. (60), (61), (78), (85), (73), (83), and (91),

$$
\begin{gathered}
\left\langle E_{\mathbf{n}}^{\sigma} E_{-\mathbf{n}}\right\rangle=\zeta_{1 \sigma}\left(\rho_{1} \sum_{\alpha} \sum_{j= \pm} \mathcal{I}_{\mathbf{k}}^{\alpha j} \delta\left(\omega+j \omega_{1 \mathbf{k}}^{\alpha}\right)+\sum_{\sigma^{\prime}} \rho_{2 \sigma^{\prime}}\left\langle E_{\mathbf{n}}^{\sigma^{\prime}} E_{-\mathbf{n}}\right\rangle\right)+\zeta_{2 \sigma} \sum_{\alpha} \sum_{j= \pm} \mathcal{I}_{\mathbf{k}}^{\alpha j} \delta\left(\omega+j \omega_{1 \mathbf{k}}^{\alpha}\right), \\
\left\langle\hat{E}_{\mathbf{n}} E_{-\mathbf{n}}\right\rangle=\frac{\rho_{1}+\sum_{\sigma} \rho_{2 \sigma} \zeta_{2 \sigma}}{1-\sum_{\sigma} \rho_{2 \sigma} \zeta_{1 \sigma}} \sum_{\alpha} \sum_{j= \pm} \mathcal{I}_{\mathbf{k}}^{\alpha j} \delta\left(\omega+j \omega_{1 \mathbf{k}}^{\alpha}\right), \\
\left\langle E_{\mathbf{n}} \hat{E}_{-\mathbf{n}}\right\rangle=\frac{\sum_{\sigma} \chi_{1 \sigma} \zeta_{1 \sigma}+\psi_{1}}{\epsilon+\psi-\sum_{\sigma} \chi_{1 \sigma} \zeta_{2 \sigma}} \sum_{\alpha} \sum_{j= \pm} \hat{\mathcal{I}}_{\mathbf{k}}^{\alpha j} \delta\left(\omega+j \omega_{2 \mathbf{k}}^{\alpha}\right), \\
\left\langle E_{\mathbf{n}} E_{-\mathbf{n}}^{\sigma}\right\rangle=\frac{1}{\epsilon+\psi} \sum_{\sigma^{\prime \prime}}\left[\chi_{1 \sigma^{\prime \prime}}+\psi_{1} \frac{\epsilon+\psi}{\epsilon+\psi-\rho_{1} \psi_{1}}\left(\frac{\rho_{1} \chi_{1 \sigma^{\prime \prime}}}{\epsilon+\psi}+\rho_{2 \sigma^{\prime \prime}}\right)\right] \sum_{\alpha} \sum_{j= \pm} \mathcal{I}_{\mathbf{k}, \sigma^{\prime \prime} \sigma}^{\alpha j} \delta\left(\omega+j \omega_{3 \mathbf{k}}^{\alpha}\right) .
\end{gathered}
$$

Expressions (95)-(97) can be inserted into (92) and (93), but (94) needs, as mentioned before, a previous definition of the plasma species. For instance, in a electron-ion plasma we should find

$$
\left\langle E_{\mathbf{n}}^{e} E_{-\mathbf{n}}\right\rangle=\frac{\left(\zeta_{1 e} \rho_{1}+\zeta_{2 e}\right)\left(1-\zeta_{1 i} \rho_{2 i}\right)+\left(\zeta_{1 i} \rho_{1}+\zeta_{2 i}\right) \zeta_{1 e} \rho_{2 i}}{\left(1-\zeta_{1 e} \rho_{2 e}\right)\left(1-\zeta_{1 i} \rho_{2 i}\right)-\zeta_{1 i} \rho_{2 e} \zeta_{1 e} \rho_{2 i}}\left\langle E_{\mathbf{n}}^{2}\right\rangle, \quad\left\langle E_{\mathbf{n}}^{i} E_{-\mathbf{n}}\right\rangle=\left\langle E_{\mathbf{n}}^{e} E_{-\mathbf{n}}\right\rangle(e \leftrightarrows i)
$$

With substitution in the kinetic equations, the result is a closed system of equations given by Eqs. (72), (77), (82), (84), (89), (90), and (92)-(94), which describes the time evolution of the system in the quasilinear approximation.

The explicit dependence on the dust charge $q$ in the kinetic equation for the dust distribution is complemented with the equation for the charging current, $I=d q / d t$, which is given by Eq. (33), and by the plasma neutrality condition,

$$
\sum_{\sigma} n_{\sigma} q_{\sigma}+n_{d} Z_{d} e=0
$$

\section{THE CASE OF A TWO-COMPONENT DUSTY PLASMA}

Let us consider the case of a dusty plasma composed by dust, electrons and a single ion species. The explicit kinetic equations for such a two-component electron-ion dusty plasma are

$$
\begin{aligned}
& \frac{\operatorname{Re}}{2}\left[\frac{\partial \epsilon\left(j \omega_{1 \mathbf{k}}^{\alpha}\right)}{\partial \omega}+\frac{\partial \psi\left(j \omega_{1 \mathbf{k}}^{\alpha}\right)}{\partial \omega}\right] \frac{\partial \mathcal{I}_{\mathbf{k}}^{\alpha j}}{\partial t}=-\operatorname{Im}\left[D_{1}\left(j \omega_{1 \mathbf{k}}^{\alpha}\right)\right] \mathcal{I}_{\mathbf{k}}^{\alpha j}, \\
& \frac{\operatorname{Re}}{2}\left[\frac{\partial D_{2}\left(j \omega_{2 \mathbf{k}}^{\alpha}\right)}{\partial \omega}\right] \frac{\partial \hat{\mathcal{I}}_{\mathbf{k}}^{\alpha j}}{\partial t}=-\operatorname{Im}\left[D_{2}\left(j \omega_{2 \mathbf{k}}^{\alpha}\right)\right] \hat{\mathcal{I}}_{\mathbf{k}}^{\alpha j}, \\
& \frac{\operatorname{Re}}{2}\left[\frac{\partial D_{3}\left(j \omega_{3 \mathbf{k}}^{\alpha}\right)}{\partial \omega}\right] \frac{\partial \mathcal{I}_{\mathbf{k}, \sigma^{\prime} \sigma}^{\alpha j}}{\partial t}=-\operatorname{Im}\left[D_{3}\left(j \omega_{3 \mathbf{k}}^{\alpha}\right)\right] \mathcal{I}_{\mathbf{k}, \sigma^{\prime} \sigma}^{\alpha j},
\end{aligned}
$$


where in each case the index $\alpha=0,1, \ldots, n$, where $n$ is the number of roots of the corresponding dispersion relation. In Eq. (102), the indexes $\sigma^{\prime} \sigma$ indicate the combinations $e e, e i$, ie, and $i i$. Moreover, $D_{1}, D_{2}$, and $D_{3}$ are given as follows:

$$
\begin{gathered}
D_{1}=\epsilon+\psi-\sum_{\sigma} \chi_{1 \sigma} \zeta_{2 \sigma}-\left(\psi_{1}+\sum_{\sigma} \chi_{1 \sigma} \zeta_{1 \sigma}\right) \frac{\rho_{1}+\sum_{\sigma^{\prime}} \rho_{2 \sigma^{\prime}} \zeta_{2 \sigma^{\prime}}}{1-\sum_{\sigma^{\prime}} \rho_{2 \sigma^{\prime}} \zeta_{1 \sigma^{\prime}}}, \\
D_{2}=1-\left(\rho_{1}+\sum_{\sigma} \rho_{2 \sigma} \zeta_{2 \sigma}\right) \frac{\sum_{\sigma^{\prime}} \chi_{1 \sigma^{\prime}} \zeta_{1 \sigma^{\prime}}+\psi_{1}}{\epsilon+\psi-\sum_{\sigma^{\prime}} \chi_{1 \sigma^{\prime}} \zeta_{2 \sigma^{\prime}}}-\sum_{\sigma} \rho_{2 \sigma} \zeta_{1 \sigma}, \\
D_{3}=\left\{1-\zeta_{1 e} \frac{\epsilon+\psi}{\epsilon+\psi-\rho_{1} \psi_{1}}\left(\frac{\rho_{1} \chi_{1 e}}{\epsilon+\psi}+\rho_{2 e}\right)-\frac{\zeta_{2 e}}{\epsilon+\psi}\left[\chi_{1 e}+\psi_{1} \frac{\epsilon+\psi}{\epsilon+\psi-\rho_{1} \psi_{1}}\left(\frac{\rho_{1} \chi_{1 e}}{\epsilon+\psi}+\rho_{2 e}\right)\right]\right\} \\
\times\left\{1-\zeta_{1 i} \frac{\epsilon+\psi}{\epsilon+\psi-\rho_{1} \psi_{1}}\left(\frac{\rho_{1} \chi_{1 i}}{\epsilon+\psi}+\rho_{2 i}\right)-\frac{\zeta_{2 i}}{\epsilon+\psi}\left[\chi_{1 i}+\psi_{1} \frac{\epsilon+\psi}{\epsilon+\psi-\rho_{1} \psi_{1}}\left(\frac{\rho_{1} \chi_{1 i}}{\epsilon+\psi}+\rho_{2 i}\right)\right]\right\} \\
-\left\{\zeta_{1 e} \frac{\epsilon+\psi}{\epsilon+\psi-\rho_{1} \psi_{1}}\left(\frac{\rho_{1} \chi_{1 i}}{\epsilon+\psi}+\rho_{2 i}\right)+\frac{\zeta_{2 e}}{\epsilon+\psi}\left[\chi_{1 i}+\psi_{1} \frac{\epsilon+\psi}{\epsilon+\psi-\rho_{1} \psi_{1}}\left(\frac{\rho_{1} \chi_{1 i}}{\epsilon+\psi}+\rho_{2 i}\right)\right]\right\} \\
\times\left\{\zeta_{1 i} \frac{\epsilon+\psi}{\epsilon+\psi-\rho_{1} \psi_{1}}\left(\frac{\rho_{1} \chi_{1 e}}{\epsilon+\psi}+\rho_{2 e}\right)+\frac{\zeta_{2 i}}{\epsilon+\psi}\left[\chi_{1 e}+\psi_{1} \frac{\epsilon+\psi}{\epsilon+\psi-\rho_{1} \psi_{1}}\left(\frac{\rho_{1} \chi_{1 e}}{\epsilon+\psi}+\rho_{2 e}\right)\right]\right\} .
\end{gathered}
$$

We find instructive to demonstrate the obtained equation (105). For the case of a two species electron-ion plasma, Eq. (88) leads to a coupled system of four equations,

$$
\begin{aligned}
& \left\langle E_{\mathbf{n}}^{e 2}\right\rangle=\zeta_{1 e} \frac{\epsilon+\psi}{\epsilon+\psi-\rho_{1} \psi_{1}}\left[\left(\frac{\rho_{1} \chi_{1 e}}{\epsilon+\psi}+\rho_{2 e}\right)\left\langle E_{\mathbf{n}}^{e 2}\right\rangle+\left(\frac{\rho_{1} \chi_{1 i}}{\epsilon+\psi}+\rho_{2 i}\right)\left\langle E_{\mathbf{n}}^{i} E_{-\mathbf{n}}^{e}\right\rangle\right] \\
& +\frac{\zeta_{2 e}}{\epsilon+\psi}\left[\chi_{1 e}\left\langle E_{\mathbf{n}}^{e 2}\right\rangle+\psi_{1} \frac{\epsilon+\psi}{\epsilon+\psi-\rho_{1} \psi_{1}}\left(\frac{\rho_{1} \chi_{1 e}}{\epsilon+\psi}+\rho_{2 e}\right)\left\langle E_{\mathbf{n}}^{e 2}\right\rangle\right] \\
& +\frac{\zeta_{2 e}}{\epsilon+\psi}\left[\chi_{1 i}\left\langle E_{\mathbf{n}}^{i} E_{-\mathbf{n}}^{e}\right\rangle+\psi_{1} \frac{\epsilon+\psi}{\epsilon+\psi-\rho_{1} \psi_{1}}\left(\frac{\rho_{1} \chi_{1 i}}{\epsilon+\psi}+\rho_{2 i}\right)\left\langle E_{\mathbf{n}}^{i} E_{-\mathbf{n}}^{e}\right\rangle\right], \\
& \left\langle E_{\mathbf{n}}^{e} E_{-\mathbf{n}}^{i}\right\rangle=\zeta_{1 e} \frac{\epsilon+\psi}{\epsilon+\psi-\rho_{1} \psi_{1}}\left[\left(\frac{\rho_{1} \chi_{1 e}}{\epsilon+\psi}+\rho_{2 e}\right)\left\langle E_{\mathbf{n}}^{e} E_{-\mathbf{n}}^{i}\right\rangle+\left(\frac{\rho_{1} \chi_{1 i}}{\epsilon+\psi}+\rho_{2 i}\right)\left\langle E_{\mathbf{n}}^{i 2}\right\rangle\right] \\
& +\frac{\zeta_{2 e}}{\epsilon+\psi}\left[\chi_{1 e}\left\langle E_{\mathbf{n}}^{e} E_{-\mathbf{n}}^{i}\right\rangle+\psi_{1} \frac{\epsilon+\psi}{\epsilon+\psi-\rho_{1} \psi_{1}}\left(\frac{\rho_{1} \chi_{1 e}}{\epsilon+\psi}+\rho_{2 e}\right)\left\langle E_{\mathbf{n}}^{e} E_{-\mathbf{n}}^{i}\right\rangle\right] \\
& +\frac{\zeta_{2 e}}{\epsilon+\psi}\left[\chi_{1 i}\left\langle E_{\mathbf{n}}^{i 2}\right\rangle+\psi_{1} \frac{\epsilon+\psi}{\epsilon+\psi-\rho_{1} \psi_{1}}\left(\frac{\rho_{1} \chi_{1 i}}{\epsilon+\psi}+\rho_{2 i}\right)\left\langle E_{\mathbf{n}}^{i 2}\right\rangle\right], \\
& \left\langle E_{\mathbf{n}}^{i} E_{-\mathbf{n}}^{e}\right\rangle=\zeta_{1 i} \frac{\epsilon+\psi}{\epsilon+\psi-\rho_{1} \psi_{1}}\left[\left(\frac{\rho_{1} \chi_{1 e}}{\epsilon+\psi}+\rho_{2 e}\right)\left\langle E_{\mathbf{n}}^{e 2}\right\rangle+\left(\frac{\rho_{1} \chi_{1 i}}{\epsilon+\psi}+\rho_{2 i}\right)\left\langle E_{\mathbf{n}}^{i} E_{-\mathbf{n}}^{e}\right\rangle\right] \\
& +\frac{\zeta_{2 i}}{\epsilon+\psi}\left[\chi_{1 e}\left\langle E_{\mathbf{n}}^{e 2}\right\rangle+\psi_{1} \frac{\epsilon+\psi}{\epsilon+\psi-\rho_{1} \psi_{1}}\left(\frac{\rho_{1} \chi_{1 e}}{\epsilon+\psi}+\rho_{2 e}\right)\left\langle E_{\mathbf{n}}^{e 2}\right\rangle\right] \\
& +\frac{\zeta_{2 i}}{\epsilon+\psi}\left[\chi_{1 i}\left(E_{\mathbf{n}}^{i} E_{-\mathbf{n}}^{e}\right\rangle+\psi_{1} \frac{\epsilon+\psi}{\epsilon+\psi-\rho_{1} \psi_{1}}\left(\frac{\rho_{1} \chi_{1 i}}{\epsilon+\psi}+\rho_{2 i}\right)\left\langle E_{\mathbf{n}}^{i} E_{-\mathbf{n}}^{e}\right\rangle\right], \\
& \left\langle E_{\mathbf{n}}^{i 2}\right\rangle=\zeta_{1 i} \frac{\epsilon+\psi}{\epsilon+\psi-\rho_{1} \psi_{1}}\left[\left(\frac{\rho_{1} \chi_{1 e}}{\epsilon+\psi}+\rho_{2 e}\right)\left\langle E_{\mathbf{n}}^{e} E_{-\mathbf{n}}^{i}\right\rangle+\left(\frac{\rho_{1} \chi_{1 i}}{\epsilon+\psi}+\rho_{2 i}\right)\left\langle E_{\mathbf{n}}^{i 2}\right\rangle\right] \\
& +\frac{\zeta_{2 i}}{\epsilon+\psi}\left[\chi_{1 e}\left\langle E_{\mathbf{n}}^{e} E_{-\mathbf{n}}^{i}\right\rangle+\psi_{1} \frac{\epsilon+\psi}{\epsilon+\psi-\rho_{1} \psi_{1}}\left(\frac{\rho_{1} \chi_{1 e}}{\epsilon+\psi}+\rho_{2 e}\right)\left\langle E_{\mathbf{n}}^{e} E_{-\mathbf{n}}^{i}\right\rangle\right] \\
& +\frac{\zeta_{2 i}}{\epsilon+\psi}\left[\chi_{1 i}\left\langle E_{\mathbf{n}}^{i 2}\right\rangle+\psi_{1} \frac{\epsilon+\psi}{\epsilon+\psi-\rho_{1} \psi_{1}}\left(\frac{\rho_{1} \chi_{1 i}}{\epsilon+\psi}+\rho_{2 i}\right)\left\langle E_{\mathbf{n}}^{i 2}\right\rangle\right] .
\end{aligned}
$$

Putting the above system in matrix homogeneous form, a $4 \times 4$ determinant is obtained from the coefficients,

$$
D_{3}=\left|\begin{array}{cccc}
D_{11} & 0 & D_{13} & 0 \\
0 & D_{11} & 0 & D_{13} \\
D_{31} & 0 & D_{33} & 0 \\
0 & D_{31} & 0 & D_{33}
\end{array}\right|=D_{11} D_{33}-D_{13} D_{31}
$$


where

$$
\begin{aligned}
& D_{11}=1-\zeta_{1 e} \frac{\epsilon+\psi}{\epsilon+\psi-\rho_{1} \psi_{1}}\left(\frac{\rho_{1} \chi_{1 e}}{\epsilon+\psi}+\rho_{2 e}\right)-\frac{\zeta_{2 e}}{\epsilon+\psi}\left[\chi_{1 e}+\psi_{1} \frac{\epsilon+\psi}{\epsilon+\psi-\rho_{1} \psi_{1}}\left(\frac{\rho_{1} \chi_{1 e}}{\epsilon+\psi}+\rho_{2 e}\right)\right], \\
& D_{13}=-\zeta_{1 e} \frac{\epsilon+\psi}{\epsilon+\psi-\rho_{1} \psi_{1}}\left(\frac{\rho_{1} \chi_{1 i}}{\epsilon+\psi}+\rho_{2 i}\right)-\frac{\zeta_{2 e}}{\epsilon+\psi}\left[\chi_{1 i}+\psi_{1} \frac{\epsilon+\psi}{\epsilon+\psi-\rho_{1} \psi_{1}}\left(\frac{\rho_{1} \chi_{1 i}}{\epsilon+\psi}+\rho_{2 i}\right)\right], \\
& D_{31}=-\zeta_{1 i} \frac{\epsilon+\psi}{\epsilon+\psi-\rho_{1} \psi_{1}}\left(\frac{\rho_{1} \chi_{1 e}}{\epsilon+\psi}+\rho_{2 e}\right)-\frac{\zeta_{2 i}}{\epsilon+\psi}\left[\chi_{1 e}+\psi_{1} \frac{\epsilon+\psi}{\epsilon+\psi-\rho_{1} \psi_{1}}\left(\frac{\rho_{1} \chi_{1 e}}{\epsilon+\psi}+\rho_{2 e}\right)\right], \\
& D_{33}=1-\zeta_{1 i} \frac{\epsilon+\psi}{\epsilon+\psi-\rho_{1} \psi_{1}}\left(\frac{\rho_{1} \chi_{1 i}}{\epsilon+\psi}+\rho_{2 i}\right)-\frac{\zeta_{2 i}}{\epsilon+\psi}\left[\chi_{1 i}+\psi_{1} \frac{\epsilon+\psi}{\epsilon+\psi-\rho_{1} \psi_{1}}\left(\frac{\rho_{1} \chi_{1 i}}{\epsilon+\psi}+\rho_{2 i}\right)\right] .
\end{aligned}
$$

The dispersion relation given by Eq. (105) corresponds to Eq. (106) equal to zero.

The connection of the kinetic equations for $f_{\sigma}$ and $f_{d}$ with the dispersion properties of the plasma may become more explicit with integration in terms of (73), (83), and (95)-(98),

$$
\begin{aligned}
\frac{\partial f_{\sigma}\left(\mathbf{v}, t^{\prime}\right)}{\partial t^{\prime}}= & -i \frac{q_{\sigma}}{m_{\sigma}} \sum_{\alpha} \sum_{j= \pm} \int d \mathbf{k} \frac{\mathbf{k}}{k} \cdot \frac{\partial}{\partial \mathbf{v}}\left(\mathbf{g}_{\mathbf{k}, j \omega_{1 \mathbf{k}}^{\alpha}}^{\alpha} \cdot \frac{\mathbf{k}}{k}\right) \mathcal{I}_{\mathbf{k}}^{\alpha j} f_{\sigma}-i \frac{q_{\sigma}}{m_{\sigma}} \sum_{\alpha} \sum_{j= \pm} \int d \mathbf{k} \frac{\mathbf{k}}{k} \cdot \frac{\partial}{\partial \mathbf{v}} \\
& \times\left[h_{\mathbf{k}, j \omega_{1 \mathbf{k}}^{\alpha}} \frac{\left(\zeta_{1 e} \rho_{1}+\zeta_{2 e}\right)\left(1-\zeta_{1 i} \rho_{2 i}\right)+\left(\zeta_{1 i} \rho_{1}+\zeta_{2 i}\right) \zeta_{1 e} \rho_{2 i}}{\left(1-\zeta_{1 e} \rho_{2 e}\right)\left(1-\zeta_{1 i} \rho_{2 i}\right)-\zeta_{1 i} \rho_{2 e} \zeta_{1 e} \rho_{2 i}}+\delta_{\sigma i}(e \leftrightarrows i)\right] \mathcal{I}_{\mathbf{k}}^{\alpha j} f_{\sigma}-i \frac{q_{\sigma}}{m_{\sigma} v_{*}} \sum_{\alpha} \sum_{j= \pm} \int d \mathbf{k} \\
& \times\left\{\left(\mathbf{g}_{\mathbf{k}, j \omega_{3 \mathbf{k}}^{\alpha}}^{\alpha} \cdot \frac{\mathbf{k}}{k}\right) \frac{1}{\epsilon+\psi} \sum_{\sigma^{\prime \prime}}\left[\chi_{1 \sigma^{\prime \prime}}+\psi_{1} \frac{\epsilon+\psi}{\epsilon+\psi-\rho_{1} \psi_{1}}\left(\frac{\rho_{1} \chi_{1 \sigma^{\prime \prime}}}{\epsilon+\psi}+\rho_{2 \sigma^{\prime \prime}}\right)\right] \mathcal{I}_{\mathbf{k}, \sigma^{\prime \prime} \sigma}^{\alpha j}+h_{\mathbf{k}, j \omega_{3 \mathbf{k}}^{\alpha}}^{\alpha} \mathcal{I}_{\mathbf{k}, \sigma \sigma}^{\alpha j}\right\} f_{\sigma}-\omega_{\sigma} f_{\sigma}, \\
& \frac{\partial f_{d}\left(\mathbf{v}, q, t^{\prime}\right)}{\partial t^{\prime}}=-i \frac{q}{m_{d}} \sum_{\alpha} \sum_{j= \pm} \int d \mathbf{k} \frac{\mathbf{k}}{k} \cdot \frac{\partial}{\partial \mathbf{v}}\left(h_{\mathbf{k}, j \omega_{1 \mathbf{k}}^{\alpha}}^{d} \frac{\rho_{1}+\sum_{\sigma} \rho_{2 \sigma} \zeta_{2 \sigma}}{1-\sum_{\sigma} \rho_{2 \sigma} \zeta_{1 \sigma}}+g_{\mathbf{k}, j \omega_{1 \mathbf{k}}^{\alpha}}^{d}\right) \mathcal{I}_{\mathbf{k}}^{\alpha j} f_{d} \\
& -i \frac{e^{2}}{m_{d} v_{*}} \sum_{\alpha} \sum_{j= \pm} \frac{\partial}{\partial q} \int d \mathbf{k}\left(h_{\mathbf{k}, j \omega_{2 \mathbf{k}}^{\alpha}}^{d}+g_{\mathbf{k}, j \omega_{2 \mathbf{k}}^{\alpha}}^{d} \frac{\sum_{\sigma} \chi_{1 \sigma} \zeta_{1 \sigma}+\psi \psi_{1}}{\epsilon+\sum_{\sigma} \chi_{1 \sigma} \zeta_{2 \sigma}}\right) \hat{\mathcal{I}}_{\mathbf{k}}^{\alpha j} f_{d}-I \frac{\partial f_{d}}{\partial q} .
\end{aligned}
$$

\section{CONCLUSIONS}

In the current work we have developed, on the basis of weak turbulence theory and starting from first principles of kinetic theory for a dusty plasma, a modified equation for the spectral energy density of the plasma electric field fluctuations and, as far as we know, two novel equations for spectral energy densities associated with the charging process of the dust particles. The analysis has been made considering the occurrence of small-scale fluctuations of the dynamical variables, so nonlinear effects are taken into account up to small order. For the present work, wave-particle interactions have been taken into account in the form of quasilinear effects and effects due to collisional charging.

In the final part of the paper, Sec. V, detailed dispersion relations have been given for electric field fluctuations and for effective field fluctuations associated to dust charge and dust current fluctuations. The time evolution equations for these fluctuations have also been presented, as well as the corresponding evolution equations for the particle distribution functions.

We conclude with the expectation that the present formalism may be useful for studies on the time evolution of turbulent dusty plasmas. Further extension of the formalism can be made, for instance, including the possibility of electromagnetic waves or the occurrence of spontaneous emission effects, similarly to what has been made for dustless plasmas $[34,35]$. Before these formal developments, however, it is of interest to produce a fullly developed analysis of the time evolution of the coupled equations of the weak turbulence theory for a dust-plasma system, including nonlinear interactions involving the electrostatic wave fields and the fluctuations of the dust charge and of the dust charging currents. Such analysis is lengthier and is the complementary extension of the present work in regard of the nonlinear interactions, with the use of Eqs. (54) and (58) in place of Eqs. (52) and (56). We believe that such fully nonlinear analysis requires a separated manuscript, which is under development and which we intend to submit for publication in the near future.

\section{ACKNOWLEDGMENT}

This work was partially supported by Brazilian agency Conselho Nacional de Desenvolvimento Científico e Tecnológico (CNPq).
[1] N. N. Rao, J. Plasma Phys. 49, 375 (1993).

[2] D. A. Mendis and M. Rosenberg, Ann. Rev. Astron. Astrophys. 32, 419 (1994).

[3] P. K. Shukla, Phys. Scr. 45, 504 (1992).
[4] U. de Angelis, Phys. Plasmas 13, 012514 (2006).

[5] R. Gaelzer, M. C. de Juli, R. S. Schneider, and L. F. Ziebell, Plasma Phys. Contr. Fusion 51, 015011 (2009).

[6] P. K. Shukla, Phys. Plasmas 7, 1044 (2000). 
[7] N. N. Rao, P. K. Shukla, and M. Y. Yu, Planet. Space Sci. 38, 543 (1990).

[8] P. K. Shukla and V. P. Silin, Phys. Scr. 45, 508 (1992).

[9] R. Z. Sagdeev and A. A. Galeev, Nonlinear Plasma Theory (Benjamin, New York, 1969).

[10] B. B. Kadomtsev, Plasma Turbulence (Academic Press, New York, 1965).

[11] R. C. Davidson, Methods in Nonlinear Plasma Theory (Academic Press, New York, 1972).

[12] V. N. Tsytovich, An Introduction to the Theory of Plasma Turbulence (Pergamon, New York, 1977).

[13] A. G. Sitenko, Fluctuations and Nonlinear Wave Interactions in Plasmas (Pergamon, New York, 1982).

[14] P. H. Yoon, Phys. Plasmas 7, 4858 (2000).

[15] P. H. Yoon, Physics of Plasmas 13, 022302 (2006).

[16] P. H. Yoon, Phys. Plasmas 14, 064504 (2007).

[17] L. Muschietti and C. T. Dum, Phys. Fluids B 3, 1968 (1991).

[18] L. F. Ziebell, R. Gaelzer, and P. H. Yoon, Phys. Plasmas 8, 3982 (2001).

[19] E. P. Kontar and H. L. Pécseli, Phys. Rev. E 65, 066408 (2002).

[20] B. Li, A. J. Willes, P. A. Robinson, and I. H. Cairns, Phys. Plasmas 10, 2748 (2003).

[21] L. F. Ziebell, R. Gaelzer, and P. H. Yoon, Phys. Plasmas 15, 032303 (2008).
[22] R. Gaelzer, L. F. Ziebell, A. F. Viñas, and P. H. Yoon, Astrophys. J. 677, 676 (2008).

[23] L. F. Ziebell, P. H. Yoon, R. Gaelzer, and J. Pavan, Plasma Phys. Contr. Fusion 54, 055012 (2012).

[24] L. F. Ziebell, P. H. Yoon, R. Gaelzer, and J. Pavan, Phys. Plasmas 21, 012306 (2014).

[25] S. Yi, C.-M. Ryu, and P. H. Yoon, Phys. Plasmas 10, 4278 (2003)

[26] S. Yi, C.-M. Ryu, and P. H. Yoon, Phys. Plasmas 11, 3191 (2004).

[27] V. N. Tsytovich and O. Havnes, Comments Plasma Phys. Control. Fusion 15, 267 (1993).

[28] P. P. J. M. Schram, A. G. Sitenko, S. A. Trigger, and A. G. Zagorodny, Phys. Rev. E 63, 016403 (2000).

[29] U. de Angelis, Phys. Scr. 45, 465 (1992).

[30] T. Matsoukas and M. Russel, J. Appl. Phys. 77, 4285 (1995).

[31] A. I. Akhiezer, I. A. Akhiezer, R. V. Polovin, A. G. Sitenko, and K. N. Stepanov, Plasma Electrodynamics: Non-linear Theory and Fluctuations (Pergamon, New York, 1975), Vol. 2.

[32] S. V. Vladimirov, Phys. Plasmas 1, 2762 (1994).

[33] V. N. Tsytovich and U. de Angelis, Phys. Plasmas 6, 1093 (1999).

[34] P. H. Yoon, Phys. Plasmas 12, 042306 (2005).

[35] R. Schlickeiser and P. H. Yoon, Phys. Plasmas 21, 092102 (2014). 